\title{
Non-commutative Donaldson-Thomas theory and vertex operators
}

\author{
KENTARO NAGAO
}

\begin{abstract}
In [26], we introduced a variant of non-commutative Donaldson-Thomas theory in a combinatorial way, which is related to the topological vertex by a wall-crossing phenomenon. In this paper, we (1) provide an alternative definition in a geometric way, (2) show that the two definitions agree with each other and (3) compute the invariants using the vertex operator method, following Okounkov, Reshetikhin and Vafa [29] and Young [12]. The stability parameter in the geometric definition determines the order of the vertex operators and hence we can understand the wall-crossing formula in non-commutative Donaldson-Thomas theory as the commutator relation of the vertex operators.
\end{abstract}

$14 \mathrm{~N} 35$

\section{Introduction}

Let $X:=\left\{x_{1} x_{2}=x_{3}{ }^{+} x_{4}{ }^{-}\right\}$be an affine toric Calabi-Yau 3-fold, which corresponds to the trapezoid with height 1 , with length $L_{+}$edge at the top and $L_{-}$at the bottom. Let $Y_{\sigma} \rightarrow X$ be a crepant resolution of $X$. Note that $Y_{\sigma}$ has $L+2$ affine lines as torus invariant closed subvarieties $\left(L:=L_{+}+L_{-}\right)$. In other words, there are $L+2$ open edges in the toric graph of $Y_{\sigma}$. Given an $(L+2)$-tuple of Young diagrams

$$
(\underline{v}, \underline{\lambda})=\left(v_{+}, v_{-}, \lambda^{(1 / 2)}, \ldots, \lambda^{(L-1 / 2)}\right) \quad\left(L:=L^{+}+L^{-}\right)
$$

associated with $L+2$ open edges (see Figure 1), we can define a torus invariant ideal sheaf $\mathcal{I}_{\underline{v}, \underline{\lambda}}$ on $Y_{\sigma}$ (Section 2.1) and a moduli space $\mathfrak{M}^{\mathrm{DT}}(\underline{\underline{\nu}}, \underline{\lambda})$ of quotient sheaves of $\mathcal{I}_{\underline{v}, \underline{\lambda}}$ (Section 4.1). Note that $\mathcal{I}_{\varnothing, \varnothing}=\mathcal{O}_{Y_{\sigma}}$ and hence the moduli space $\mathfrak{M}^{\mathrm{DT}}(\underline{\varnothing}, \varnothing)$ is the Hilbert scheme of closed subschemes of $Y_{\sigma}$. We define Euler characteristic version of open (commutative) Donaldson-Thomas invariants by the Euler characteristics of the connected components of $\mathfrak{M}^{\mathrm{DT}}(\underline{\nu}, \underline{\lambda})^{1}$. The torus action of $Y_{\sigma}$ induces the torus action on $\mathfrak{M}^{\mathrm{DT}}(\underline{\underline{\nu}}, \underline{\lambda})$. The torus fixed point set is isolated and parametrized in

${ }^{1}$ The word "open" stems from such terminologies as "open topological string theory". According to Aganagic, Klemm, Mariño and Vafa [1], open topological string partition function is given by summing up the generating functions of these invariants over Young diagrams. 
terms of $L$-tuples of 3-dimensional Young diagrams. Thus the generating function of the open Donaldson-Thomas invariants can be described in terms of topological vertex (see Aganagic, Klemm, Mariño and Vafa [1], Maulik, Nekrasov, Okounkov and Pandharipande [21], and Section 4.2).

Let $A_{\sigma}$ be a non-commutative crepant resolution of the affine toric Calabi-Yau 3fold $X$. We can identify the derived category of coherent sheaves on $Y_{\sigma}$ and the one of $A_{\sigma}$-modules by a derived equivalence. A parameter $\zeta$ gives a Bridgeland's stability condition of this derived category, and hence a core $\mathcal{A}^{\zeta}$ of a t-structure on it (Definition 1.7). In fact, we have two specific parameters such that the corresponding t-structures coincide with the ones given by $Y_{\sigma}$ or $A_{\sigma}$ respectively. Given an element in $\mathcal{A}^{\zeta}$, we can restrict it to get a sheaf on the smooth locus $X^{\mathrm{sm}}$. Since the singular locus $X^{\text {sing }}$ is compact, it makes sense to study those elements in $\mathcal{A}^{\zeta}$ which are isomorphic to $\mathcal{I}_{\underline{v}, \underline{\lambda}}$ outside a compact subset of $X$, or in other words, those elements in $\mathcal{A}^{\zeta}$ which have the same asymptotic behavior as $\mathcal{I}_{\underline{\underline{\nu}}, \underline{\lambda}}$. We will study the moduli spaces of such objects as noncommutative analogues of $\mathfrak{M}^{\mathrm{DT}}(\underline{\underline{\nu}}, \underline{\lambda})$. In general the ideal sheaf $\mathcal{I}_{\underline{\underline{v}}, \underline{\lambda}}$ is not an element in $\mathcal{A}^{\zeta}$, however $P_{\underline{\nu}, \underline{\lambda}}^{\zeta}:=H_{\mathcal{A}^{\zeta}}^{0}\left(\mathcal{I}_{\underline{\underline{\nu}}, \underline{\lambda}}\right)$ is always in $\mathcal{A}^{\zeta}$. We will construct the moduli space $\mathfrak{M}^{\mathrm{ncDT}}(\zeta, \underline{\nu}, \underline{\lambda})$ of quotients of $P_{\underline{\underline{\nu}}, \underline{\lambda}}^{\zeta}$ in $\mathcal{A}^{\zeta}$ as a GIT quotient (Section 5.1). Note that $\mathfrak{M}^{\mathrm{ncDT}}(\zeta, \varnothing, \varnothing)$ is the moduli space we have studied in [24]. We define Euler characteristic version of open non-commutative Donaldson-Thomas invariants by the Euler characteristics of the connected components of $\mathfrak{M}^{\mathrm{ncDT}}(\zeta, \underline{\nu}, \underline{\lambda})^{2}$.

The torus action on $Y_{\sigma}$ induces a torus action on the moduli $\mathfrak{M}^{\mathrm{ncDT}}(\zeta, \underline{\nu}, \underline{\lambda})$. We will compute the Euler characteristic by counting the number of torus fixed points. For a generic $\zeta$, the core $\mathcal{A}^{\zeta}$ of the t-structure is isomorphic to the category of $A_{\sigma}^{\zeta}$-modules, where $A_{\sigma}^{\zeta}$ is associated with a quiver with a potential. Hence, we can describe the torus fixed point set on $\mathfrak{M}^{\text {ncDT }}(\zeta, \underline{v}, \underline{\lambda})$ in terms of a crystal melting model (see Okounkov, Reshetikhin and Vafa [29] and Ooguri and Yamazaki [30]), which we have studied in [26]. In fact, a particle in the grand state crystal gives a weight vector in $P_{\underline{v}, \underline{\lambda}}^{\zeta}$ with respect to the torus action, and a crystal obtained by removing a finite number of particles from the grand state crystal gives a torus fixed point in $\mathfrak{M}^{\mathrm{ncDT}}(\zeta, \underline{\nu}, \underline{\lambda})$ (Section 3.1). The invariants in this paper agree with the ones defined in [26].

Finally, we provide explicit formulas for the generating functions of the Euler characteristic version of the open commutative and non-commutative Donaldson-Thomas invariants using vertex operator method, following Okounkov, Reshetikhin and Vafa [29], Young [12] and Bryan and Young [11] ${ }^{3}$. The order of the vertex operators is determined

${ }^{2}$ The reader may also refer to [28] in which we study the invariants in the physics context.

${ }^{3}$ During preparing this paper, the author was informed that Piotr Sulkowski [32] and Benjamin Young [34] provide similar computations independently. 
by the chamber in which the parameter $\zeta$ is. Hence we can understand the wall-crossing formula as the commutator relation of the vertex operators.

In Szendroi's original non-commutative Donaldson-Thomas theory [33] the moduli spaces admit symmetric obstruction theory and the invariants are defined as the virtual counting of the moduli spaces in the sense of Behrend-Fantechi $[3]^{4}$. In the case when $v_{+}=v_{-}=\varnothing$, we show that the moduli space $\mathfrak{M}^{\mathrm{ncDT}}(\zeta, \underline{\varnothing}, \underline{\lambda})$ admits a symmetric obstruction theory (Section 5.2). Using the result in [4], we can verify that the virtual counting coincide with the (non-weighted) Euler characteristics up to signs as in Szendroi [33], Mozgovoy and Reineke [23], and the author and Nakajima [27; 24] (see Section 6.1). We can also compute the generating function of the weighted (or non-weighted) Euler characteristics using Joyce-Song's theory [20; 19].

The plan of this paper is as follows: Section 1 contains basic observations on the core $\mathcal{A}^{\xi}$ of the t-structure of the derived category. In Section 2, the definition of Euler characteristic version of open non-commutative Donaldson-Thomas invariants is provided. Then, we compute the generating function using vertex operators in Section 3. Finally, we study open Donaldson-Thomas invariants and topological vertex as "limits" of open non-commutative Donaldson-Thomas invariants in Section 4. Section 2, Section 3 and Section 4 are the main parts of this paper. In Section 5 we construct the moduli spaces used in Section 2 and 4 to define invariants. Moreover, we construct symmetric obstruction theory on the moduli space in the case of $v_{+}=v_{-}=\varnothing$ in Section 5.2. The relation between weighted Euler characteristic and Euler characteristic is discussed in Section 6.1. Throughout this paper we work on the half of the whole space of stability parameters. We will have a discussion about the other half of the stability space in Section 6.2. The computation in Section 3 depends on an explicit combinatorial description of the derived equivalence. We leave it until Section 6.3 since it is very technical.

\section{Acknowledgements}

The author is supported by JSPS Fellowships for Young Scientists (number 19-2672). He thanks to Jim Bryan for letting him know the result of [11] and recommending him to apply the vertex operator method in the setting of this paper. He also thanks to Osamu Iyama, Hiroaki Kanno, Hiraku Nakajima, Piotr Sulkowski, Yukinobu Toda, Masahito Yamazaki and Benjamin Young for useful comments.

This paper was written while the author is visiting the University of Oxford. He is grateful to Dominic Joyce for the invitation and to the Mathematical Institute for hospitality.

\footnotetext{
${ }^{4}$ Virtual counting coincides with the weighted Euler characteristic weighted by the Behrend function.
} 


\section{Notations}

Let $\mathbb{Z}_{\mathrm{h}}$ denote the set of half integers and $L$ be a positive integer. We set $I:=\mathbb{Z} / L \mathbb{Z}$ and $I_{\mathrm{h}}:=\mathbb{Z}_{\mathrm{h}} / L \mathbb{Z}$. The two natural projections $\mathbb{Z} \rightarrow I$ and $\mathbb{Z}_{\mathrm{h}} \rightarrow I_{\mathrm{h}}$ are denoted by the same symbol $\pi$. We sometimes identify $I$ and $I_{\mathrm{h}}$ with $\{0, \ldots, L-1\}$ and $\{1 / 2, \ldots, L-1 / 2\}$ respectively. The symbols $n, h, i$ and $j$ are used for elements in $\mathbb{Z}, \mathbb{Z}_{\mathrm{h}}, I$ and $I_{\mathrm{h}}$ respectively.

Throughout this paper, the following data play crucial roles:

- a map $\sigma: \mathbb{Z}_{\mathrm{h}} \rightarrow\{ \pm\}$, which determines the crepant resolution $Y_{\sigma} \rightarrow X$ and the non-commutative crepant resolution $A_{\sigma}$,

- a pair of Young diagram $\underline{v}=\left(v_{+}, v_{-}\right)$and an $L$-tuple of Young diagrams $\underline{\lambda}=\left(\lambda^{(1 / 2)}, \ldots, \lambda^{(L-1 / 2)}\right)$, which determines the "asymptotic behaviors" of (complexes of) sheaves we will count,

- a stability parameter $\zeta$, which determines the t-structure where we will work on, and

- a bijection $\theta: \mathbb{Z}_{\mathrm{h}} \rightarrow \mathbb{Z}_{\mathrm{h}}$, which determines the chamber where the stability parameter $\zeta$ is.

We sometimes identify a Young diagram $\mu$ with a map $\mu: \mathbb{Z}_{\mathrm{h}} \rightarrow\{ \pm\}$ such that $\mu(h)=-$ for $h \ll 0$ and $\mu(h)=+$ for $h \gg 0^{5}$. We identify an $L$-tuple of Young diagrams $\underline{\lambda}$ with a map $\underline{\lambda}: \mathbb{Z}_{\mathrm{h}} \rightarrow\{ \pm\}$ by

$$
\underline{\lambda}(h)=\lambda^{(\pi(h))}\left(\frac{h-\pi(h)}{L}+\frac{1}{2}\right) .
$$

We define the following categories:

$\operatorname{Coh}\left(Y_{\sigma}\right):$ the Abelian category of coherent sheaves on $Y_{\sigma}$,

$\operatorname{Coh}_{\text {cpt }}\left(Y_{\sigma}\right)$ : the full subcategory of $\operatorname{Coh}\left(Y_{\sigma}\right)$ consisting of coherent sheaves with compact supports,

$D^{b} \operatorname{Coh}\left(Y_{\sigma}\right)$ : the bounded derived category $\operatorname{Coh}\left(Y_{\sigma}\right)$,

$D_{\mathrm{cpt}}^{b} \operatorname{Coh}\left(Y_{\sigma}\right)$ : the full subcategory of $D^{b} \operatorname{Coh}\left(Y_{\sigma}\right)$ consisting of complexes with compactly supported cohomologies,

$\bmod A_{\sigma}:$ the Abelian category of finitely generated left $A_{\sigma}$-modules,

${ }^{5}$ Such a map $\mu$ is called a Maya diagram. See, for example, the author's paper [25, Section 2] for the correspondence between a Young diagram and a Maya diagram. 
$\bmod _{\text {fin }} A_{\sigma}$ : the full subcategory of $\bmod A_{\sigma}$ consisting of finite dimensional modules,

$D^{b}\left(\bmod A_{\sigma}\right):$ the bounded derived category of $\bmod A_{\sigma}$,

$D_{\text {fin }}^{b}\left(\bmod A_{\sigma}\right)$ : the full subcategory of $D^{b}\left(\bmod A_{\sigma}\right)$ consisting of complexes with finite dimensional cohomologies.

\section{T-structure and chamber structure}

\subsection{Non-commutative and commutative crepant resolutions}

Let $\sigma$ be a map from $I_{\mathrm{h}}$ to $\{ \pm\}$. In [24], following Hanany and Vegh [15], we introduced a quiver with a potential $A_{\sigma}=\left(Q_{\sigma}, w_{\sigma}\right)$, which is a non-commutative crepant resolution of $X$. First, we set

$$
\begin{aligned}
H(\sigma) & :=\{n \in \mathbb{Z} \mid \sigma(n-1 / 2)=\sigma(n+1 / 2)\}, & & I_{H}(\sigma):=\pi(H(\sigma)), \\
S(\sigma) & :=\{n \in \mathbb{Z} \mid \sigma(n-1 / 2) \neq \sigma(n+1 / 2)\}, & & I_{S}(\sigma):=\pi(S(\sigma)) .
\end{aligned}
$$

The symbol $H$ and $S$ represent "hexagon" and "square" respectively. We use such notations since an element in each set corresponds to a hexagon or a square in the dimer model (see [24, Section 1.2]). The vertices of $Q_{\sigma}$ are parametrized by $I$ and the arrows are given by

$$
\left(\bigsqcup_{j \in I_{\mathrm{h}}} h_{j}^{+}\right) \sqcup\left(\bigsqcup_{j \in I_{\mathrm{h}}} h_{j}^{-}\right) \sqcup\left(\bigsqcup_{i \in I_{H}(\sigma)} r_{i}\right) .
$$

Here $h_{j}^{+}$(respectively $h_{j}^{-}$) is an edge from $j-1 / 2$ to $j+1 / 2$ (respectively from $j+1 / 2$ to $j-1 / 2$ ) and $r_{i}$ is an edge from $i$ to itself. See [24, Section 1.2] for the definition of the potential $w_{\sigma}$.

Let $P_{i}$ (respectively $S_{i}$ ) be the projective (respectively simple) $A_{\sigma}$-module corresponding to the vertex $i$. Let $K_{\text {num }}\left(\bmod _{\text {fin }} A_{\sigma}\right)$ be the numerical Grothendieck group of $\bmod _{\mathrm{fin}} A_{\sigma}$, which we identify with $\mathbb{Z}^{I}$ by the natural basis $\left\{\left[S_{i}\right]\right\}$. We put $\delta=(1, \ldots, 1) \in K_{\text {num }}\left(\bmod _{\text {fin }} A_{\sigma}\right)$.

We identity the dual space $\left(K_{\text {num }}\left(\bmod _{\text {fin }} A_{\sigma}\right) \otimes \mathbb{R}\right)^{*}$ with $\mathbb{R}^{I}$ by the dual basis of $\left\{\left[S_{i}\right]\right\}$. Take $\zeta_{\text {cyc }}^{\circ}:=(-L+1,1,1, \ldots, 1) \in \mathbb{R}^{I}$.

Theorem 1.1 (Ishii and Ueda [18]) The moduli space of $\zeta_{\mathrm{cyc}}^{\circ}-$ stable $\left(=\zeta_{\mathrm{cyc}}^{\circ}-\right.$ semistable) $A_{\sigma}$-modules with dimension vectors $=\delta$ gives a crepant resolution of $X$. 
Let $Y_{\sigma}$ denote this crepant resolution.

Theorem 1.2 ([24, Section 1$]^{6}$, see Section 4.1) We have a derived equivalence between $D^{b} \operatorname{Coh}\left(Y_{\sigma}\right)$ and $D^{b}\left(\bmod A_{\sigma}\right)$, which restricts to an equivalence between $D_{\text {cpt }}^{b} \operatorname{Coh}\left(Y_{\sigma}\right)\left(\right.$ respectively $\left.{ }^{-1} \operatorname{Per}(Y / X)\right)$ and $D_{\text {fin }}^{b}\left(\bmod A_{\sigma}\right)\left(\operatorname{respectively~} \bmod A_{\sigma}\right)$.

\subsection{Stability condition and tilting}

For $\zeta^{\circ} \in\left(K_{\text {num }}\left(\bmod _{\text {fin }} A_{\sigma}\right) \otimes \mathbb{R}\right)^{*} \simeq \mathbb{R}^{I}$ such that $\zeta^{\circ} \cdot \delta=0$, we define the group homomorphism

$$
Z_{\zeta^{\circ}}: K_{\text {num }}\left(\bmod _{\text {fin }} A_{\sigma}\right) \rightarrow \mathbb{C}
$$

by

$$
Z_{\zeta^{\circ}}(\mathbf{v}):=\left(-\zeta^{\circ}+\eta \sqrt{-1}\right) \cdot \mathbf{v}
$$

where $\eta=(1, \ldots, 1) \in\left(K_{\text {num }}\left(\bmod _{\mathrm{fin}} A_{\sigma}\right) \otimes \mathbb{R}\right)^{*} \simeq \mathbb{R}^{I}$. Then $\left(\bmod _{\mathrm{fin}} A_{\sigma}, Z_{\zeta^{\circ}}\right)$ gives a stability condition on $D_{\text {fin }}^{b}\left(\bmod A_{\sigma}\right)$ in the sense of Bridgeland [9].

For a pair of real numbers $t_{1}>t_{2}$, let $\mathcal{D}_{\text {fin }}^{\zeta^{\circ}}\left[t_{1}, t_{2}\right)$ be the full subcategory of $D_{\text {fin }}^{b}\left(\bmod A_{\sigma}\right)$ consisting of elements whose Harder-Narasimhan factors have phases less or equal to $t_{1} \pi$ and larger than $t_{2} \pi$. The following claims are standard (see [9]):

Lemma 1.3 (1) $\mathcal{D}_{\text {fin }}^{\zeta^{\circ}}\left[t_{1}+1, t_{2}+1\right)=\mathcal{D}_{\text {fin }}^{\zeta^{\circ}}\left[t_{1}, t_{2}\right)[1]$ where [1] represents the shift in the derived category.

(2) $\mathcal{D}_{\text {fin }}^{\zeta^{\circ}}[t, t-1)$ is a core of a $t$-structure for any $t$.

(3) $\mathcal{D}_{\text {fin }}^{\zeta^{\circ}}[1,0)=\bmod _{\text {fin }} A_{\sigma}$.

(4) For $t>s>t-1$, the pair of subcategories

$$
\left(\mathcal{D}_{\text {fin }}^{\zeta^{\circ}}[t, s), \mathcal{D}_{\text {fin }}^{\zeta^{\circ}}[s, t-1)\right)
$$

gives a torsion pair (see Bridgeland [8, Definition 2.4]) for the Abelian category $\mathcal{D}_{\text {fin }}^{\zeta^{\circ}}[t, t-1)$.

\footnotetext{
${ }^{6}$ It is known by Mozgovoy [22] and Bocklandt [7] that a quiver with a potential given from a brane tiling satisfying the "consistency condition" (see Mozgovoy and Reineke [23], Davison [14], Broomhead [10] and Ishii and Ueda [17]) is a non-commutative crepant resolution over its center (see van den Bergh [5]). The claim of this theorem is a little bit stronger, that is, $A_{\sigma}$ is given by the construction in van den Bergh [6] and hence we have ${ }^{-1} \operatorname{Per}(Y / X) \simeq \bmod A_{\sigma}$. We will use this equivalence of the Abelian categories in Section 4.
} 
(5) For $t>s>t-1, \mathcal{D}_{\text {fin }}^{\zeta^{\circ}}[s, s-1)$ is obtained from $\mathcal{D}_{\text {fin }}^{\zeta^{\circ}}[t, t-1)$ by tilting with respect to the torsion pair above (see Happel, Reiten and Smalø [16] and Bridgeland [8, Proposition 2.5]), that is,

$$
\begin{aligned}
& \mathcal{D}_{\text {fin }}^{\zeta^{\circ}}[s, s-1)= \\
& \quad\left\{E \in D_{\text {fin }}^{b}\left(\bmod A_{\sigma}\right) \mid H_{\mathcal{D}_{\text {fin }}^{\zeta^{\circ}}[t, t-1)}^{0}(E) \in \mathcal{D}_{\text {fin }}^{\zeta^{\circ}}[s, t-1), H_{\mathcal{D}_{\text {fin }}^{\zeta^{\circ}}[t, t-1)}^{1}(E) \in \mathcal{D}_{\text {fin }}^{\zeta^{\circ}}[t, s)\right\}, \\
& \mathcal{D}_{\text {fin }}^{\zeta^{\circ}}[t, t-1)= \\
& \quad\left\{E \in D_{\text {fin }}^{b}\left(\bmod A_{\sigma}\right) \mid H_{\mathcal{D}_{\text {fin }}^{\zeta^{\circ}}[s, s-1)}^{0}(E) \in \mathcal{D}_{\text {fin }}^{\zeta^{\circ}}[s, t-1), H_{\mathcal{D}_{\text {fin }}^{\zeta^{\circ}}[s, s-1)}^{-1}(E) \in \mathcal{D}_{\text {fin }}^{\zeta^{\circ}}[t-1, s-1)\right\},
\end{aligned}
$$

where

$$
H_{\mathcal{D}_{\text {fin }}^{\xi^{\circ}}[t, t-1)}^{(-)}
$$

represents the cohomology with respect to the $t$-structure corresponding to $\mathcal{D}_{\text {fin }}^{\zeta^{\circ}}[t, t-1)$.

Lemma 1.4 The algebra $A_{\sigma}$ is (left) Noetherian.

Proof In [24], it is shown that $A_{\sigma}$ is isomorphic to $f_{*}$ End $V$ for a vector bundle $V$ on $Y_{\sigma}$, where $f$ is the contraction $Y_{\sigma} \rightarrow X$. Since $f$ is proper, $A_{\sigma}$ is finitely generated as an $\mathcal{O}_{X}$-module. Hence $A_{\sigma}$ is Noetherian.

Proposition 1.5 For $0<t<1$ we put

$$
\mathcal{D}_{\text {fin }}^{\zeta^{\circ}}[1, t)^{\perp}:=\left\{E \in \bmod A_{\sigma} \mid \operatorname{Hom}_{A_{\sigma}}(F, E)=0 \text {, for all } F \in \mathcal{D}_{\text {fin }}^{\zeta^{\circ}}[1, t)\right\} .
$$

Then the pair of full subcategory $\left(\mathcal{D}_{\text {fin }}^{\zeta^{\circ}}[1, t), \mathcal{D}_{\text {fin }}^{\zeta^{\circ}}[1, t)^{\perp}\right)$ gives a torsion pair in $\bmod A_{\sigma}$.

Proof We will prove that every object $F \in \bmod A_{\sigma}$ fits into a short exact sequence

$$
0 \rightarrow E \rightarrow F \rightarrow G \rightarrow 0
$$

for some pair of objects $E \in \mathcal{D}_{\text {fin }}^{\zeta^{\circ}}[1, t)$ and $G \in \mathcal{D}_{\text {fin }}^{\zeta^{\circ}}[1, t)^{\perp}$.

By Lemma 1.4, $F$ has the maximal finite dimensional submodule $F^{(1)}$. Let $F^{(2)}$ denote the cokernel of the inclusion $F^{(1)} \hookrightarrow F$. Note that $\operatorname{Hom}_{A_{\sigma}}\left(X, F^{(2)}\right)=0$ for any finite dimensional $A_{\sigma}$-module $X$.

Let

$$
0 \rightarrow F^{(3)} \rightarrow F^{(1)} \rightarrow F^{(4)} \rightarrow 0
$$

be the exact sequence such that $F^{(3)} \in \mathcal{D}_{\text {fin }}^{\zeta^{\circ}}[1, t)$ and $F^{(4)} \in \mathcal{D}_{\text {fin }}^{\zeta^{\circ}}[t, 0)$. Note that for any $X \in \mathcal{D}_{\text {fin }}^{\zeta^{\circ}}[1, t)$ we have $\operatorname{Hom}_{A_{\sigma}}\left(X, F^{(4)}\right)=0$. 
Let $F^{(5)}$ denote the cokernel of the inclusion $F^{(3)} \hookrightarrow F$. Then we have the following exact sequence:

$$
0 \rightarrow F^{(4)} \rightarrow F^{(5)} \rightarrow F^{(2)} \rightarrow 0 .
$$

This implies $\operatorname{Hom}_{A_{\sigma}}\left(X, F^{(5)}\right)=0$ for any $X \in \mathcal{D}_{\text {fin }}^{\zeta^{\circ}}[1, t)$. Put $E:=F^{(3)}$ and $G:=$ $F^{(5)}$, then the claim follows.

Definition 1.6 For $0<t<1$ let $\mathcal{D}^{\xi^{\circ}}[t, t-1)$ denote the core of the t-structure given from $\bmod A_{\sigma}$ by tilting with respect to the torsion pair in Proposition 1.5, that is, $\mathcal{D}^{\zeta^{\circ}}[t, t-1)=\left\{E \in D^{b}\left(\bmod A_{\sigma}\right) \mid H_{\bmod A_{\sigma}}^{0}(E) \in \mathcal{D}_{\text {fin }}^{\zeta^{\circ}}[1, t)^{\perp}, H_{\bmod A_{\sigma}}^{1}(E) \in \mathcal{D}_{\text {fin }}^{\zeta^{\circ}}[1, t)\right\}$.

We have the following bijection:

$$
\begin{aligned}
\left\{\left(\zeta^{\circ}, T\right) \mid \zeta^{\circ} \cdot \delta=0,\right. & T \in \mathbb{R}\} \stackrel{\sim}{\longrightarrow} \mathbb{R}^{I} \simeq\left(K_{\mathrm{num}}\left(\bmod _{\mathrm{fin}} A_{\sigma}\right) \otimes \mathbb{R}\right)^{*} \\
\left(\zeta^{\circ}, T\right) & \longmapsto \zeta^{\circ}-T \eta .
\end{aligned}
$$

The inverse map is given by

$$
T:=-\zeta \cdot \eta / L, \quad \zeta^{\circ}:=\zeta+T \eta
$$

for $\zeta \in\left(K_{\text {num }}\left(\bmod _{\text {fin }} A_{\sigma}\right) \otimes \mathbb{R}\right)^{*}$. For a fixed $T$, take $0<t<1$ such that $\tan (t \pi)=1 / T$. Note that for an element $V \in \bmod _{\text {fin }} A_{\sigma}$ we have

$$
\phi_{Z_{\zeta^{\circ}}}(V)<t \pi \Longleftrightarrow \zeta([V])<0
$$

where $\phi_{Z_{\zeta^{\circ}}}(V):=\arg \left(Z_{\zeta^{\circ}}([V])\right)$.

\section{Definition 1.7}

$$
\mathcal{A}_{\text {fin }}^{\zeta}:=\mathcal{D}_{\text {fin }}^{\zeta^{\circ}}[t, t-1), \quad \quad \mathcal{A}^{\zeta}:=\mathcal{D}^{\zeta^{\circ}}[t, t-1) .
$$

Remark 1.8 We have the natural action rot of $\mathbb{R}$ on the space of Bridgeland's stability conditions given by rotation of the complex line which is the target of the central charge. We can embed $\left(K_{\text {num }}\left(\bmod _{\text {fin }} A_{\sigma}\right) \otimes \mathbb{R}\right) *$ into the space of Bridgeland's stability conditions by

$$
\zeta \mapsto Z_{\zeta}:=\operatorname{rot}_{t}\left(Z_{\zeta^{\circ}}\right)
$$

Note that $\mathcal{D}_{\text {fin }}^{\zeta^{\circ}}[t, t-1)$ agrees with $\mathcal{D}_{\text {fin }}^{\zeta}[1,0)$. This is the reason why we call $\zeta$ a stability parameter, although we will use the former description since it is more convenient in our argument. 


\subsection{Chamber structure}

A stability parameter $\zeta$ is said to be generic if there is no $Z_{\zeta^{\circ}}-$ semistable objects with phase $t$. Then we get a chamber structure in $\left(K_{\text {num }}\left(\bmod _{\text {fin }} A_{\sigma}\right) \otimes \mathbb{R}\right)^{*} \simeq \mathbb{R}^{I}$.

Proposition 1.9 The chamber structure coincides with the affine root chamber structure of type $A_{L-1}$.

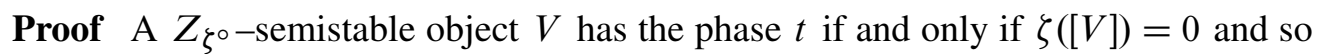
the genericity in this paper agrees with the one in [24]. Then the claim follows from [24, Proposition 2.10, Corollary 2.12].

Here we give a brief review for the affine root system of type $A_{L-1}$. We call $P:=$ $K_{\text {num }}\left(\bmod _{\mathrm{fin}} A_{\sigma}\right)$ the root lattice and $\alpha_{i}:=\left[S_{i}\right] \in P$ a simple root. For $h, h^{\prime} \in \mathbb{Z}_{\mathrm{h}}$, we define $\alpha_{\left[h, h^{\prime}\right]} \in P$ by

$$
\alpha_{\left[h, h^{\prime}\right]}:= \begin{cases}0 & h=h^{\prime}, \\ \alpha_{\pi(h+1 / 2)}+\cdots+\alpha_{\pi\left(h^{\prime}-1 / 2\right)} & h<h^{\prime}, \\ -\alpha_{\pi(h-1 / 2)}-\cdots-\alpha_{\pi\left(h^{\prime}+1 / 2\right)} & h>h^{\prime}\end{cases}
$$

and $\delta:=\alpha_{0}+\cdots+\alpha_{L-1}$. We set

$$
\Lambda:=\left\{\alpha_{\left[h, h^{\prime}\right]} \mid h \neq h^{\prime}\right\}, \quad \Lambda^{+}:=\left\{\alpha_{\left[h, h^{\prime}\right]} \mid h<h^{\prime}\right\}, \quad \Lambda^{-}:=\left\{\alpha_{\left[h, h^{\prime}\right]} \mid h>h^{\prime}\right\}
$$

and

$$
\Lambda^{\mathrm{re}}:=\left\{\alpha_{\left[h, h^{\prime}\right]} \mid h \not \equiv h^{\prime}(\bmod L)\right\}, \quad \Lambda^{\mathrm{im}}:=\{m \delta \mid m \neq 0\} .
$$

An element in $\Lambda$ (respectively $\Lambda^{+}, \Lambda^{-}, \Lambda^{\mathrm{re}}, \Lambda^{\mathrm{im}}$ ) is called a root (respectively positive root, negative root, real root, imaginary root). Note that $\Lambda=\Lambda^{+} \sqcup \Lambda^{-}=\Lambda^{\text {re }} \sqcup \Lambda^{\mathrm{im}}$. We put $\Lambda^{\text {re,+ }}:=\Lambda^{\text {re }} \cap \Lambda^{+}$and define $\Lambda^{\text {re,--}}, \Lambda^{\mathrm{im},+}$ and $\Lambda^{\mathrm{im},-}$ in the same way.

For a root $\alpha$, let $W_{\alpha}$ denote the hyperplane in $\left(K_{\text {num }}\left(\bmod _{\text {fin }} A_{\sigma}\right) \otimes \mathbb{R}\right)^{*}$ given by

$$
W_{\alpha}:=\left\{\zeta \in\left(K_{\text {num }}\left(\bmod _{\text {fin }} A_{\sigma}\right) \otimes \mathbb{R}\right)^{*} \mid \zeta \cdot \alpha=0\right\} .
$$

The walls in the affine root chamber structure of type $A_{L-1}$ is given by

$$
W_{\delta} \cup \bigcup_{\alpha \in \Lambda^{\mathrm{re},+}} W_{\alpha} .
$$

Throughout this paper, we work on the area below the wall $W_{\delta}$, that is, on the area $\{\zeta \mid \zeta \cdot \delta<0\}$. 


\subsection{Parametrization of chambers}

Let $\Theta$ denote the set of bijections $\theta: \mathbb{Z}_{\mathrm{h}} \rightarrow \mathbb{Z}_{\mathrm{h}}$ such that

- $\theta(h+L)=\theta(h)+L$ for any $h \in \mathbb{Z}_{\mathrm{h}}$, and

- $\theta(1 / 2)+\cdots+\theta(L-1 / 2)=1 / 2+\cdots+(L-1 / 2)=L^{2} / 2$.

We have a natural bijection between $\Theta$ and the set of chambers in the area $\{\zeta \mid \zeta \cdot \delta<0\}$. An element $\zeta_{\theta}$ in the chamber $C_{\theta}$ corresponding to $\theta \in \Theta$ satisfies the following condition:

$$
\alpha_{\left[h, h^{\prime}\right]} \cdot \zeta_{\theta}<0 \Longleftrightarrow \theta(h)<\theta\left(h^{\prime}\right)
$$

for any $h<h^{\prime}$. For $\theta \in \Theta$ and $i \in I$, we define $\alpha(\theta, i) \in P$ by

$$
\alpha(\theta, i):=\alpha_{[\theta(n-1 / 2), \theta(n+1 / 2)]} \quad(\pi(n)=i) .
$$

Then the chamber $C_{\theta}$ is adjacent to the walls $W_{\alpha(\theta, i)}$ and we have $\zeta_{\theta} \cdot \alpha(\theta, i)<0$ for $\zeta_{\theta} \in C_{\theta}$.

Let $\theta_{i}: \mathbb{Z}_{\mathrm{h}} \rightarrow \mathbb{Z}_{\mathrm{h}}$ be the bijection given by

$$
\theta_{i}(h)= \begin{cases}h+1 & \pi(h+1 / 2)=i, \\ h-1 & \pi(h-1 / 2)=i, \\ h & \text { otherwise }\end{cases}
$$

Then we have $\alpha(\theta, i)=-\alpha\left(\theta \circ \theta_{i}, i\right)$ and the chambers $C_{\theta}$ and $C_{\theta \circ \theta_{i}}$ are separated by the wall $W_{\alpha(\theta, i)}=W_{\alpha\left(\theta \circ \theta_{i}, i\right)}$.

\subsection{Mutation}

Assume that $T<0$ and $\zeta^{\circ}$ is such that $\left(\zeta^{\circ}, T^{\prime}\right)$ is not on an intersection of two walls for any $T^{\prime} \in \mathbb{R}$. Let $\left\{T_{r}\right\}\left(T_{1}<T_{2}<\cdots<0\right)$ be the set of all the parameters $T_{r}<0$ such that $\left(\zeta^{\circ}, T_{r}\right)$ is not generic. According to the argument at the end of the previous subsection, we have the sequence $\left\{i_{r}\right\}$ of elements in $I$ such that $\left(\zeta^{\circ}, T_{r}\right)$ for any $r$ is on the wall $W_{\alpha_{r}}$ for

$$
\alpha_{r}:=\alpha\left(\theta_{i_{1}} \circ \cdots \circ \theta_{i_{r-1}}, i_{r}\right) .
$$

Take the minimal positive integer $R$ such that $T<T_{R}$ and put $A_{\sigma}^{\zeta}:=A_{\sigma \circ \theta_{i_{1}} \circ \cdots \circ \theta_{i_{R-1}}}$. Using this notation we have the following equivalencies of the Abelian categories:

Proposition 1.10

$$
\mathcal{A}_{\text {fin }}^{\zeta} \simeq \bmod _{\text {fin }} A_{\sigma}^{\zeta}, \quad \mathcal{A}^{\zeta} \simeq \bmod A_{\sigma}^{\zeta} .
$$


Proof We have the derived equivalence between $A_{\sigma \circ \theta_{i_{1}} \circ \cdots \circ \theta_{i_{r-1}}}$ and $A_{\sigma \circ \theta_{i_{1}} \circ \cdots \circ \theta_{i_{r}}}$ obtained by the tilting generator as in [24, Proposition 3.1]. It is easy to see that, under this equivalence, the module category of $A_{\sigma \circ \theta_{i_{1}} \circ \cdots \circ \theta_{i_{r}}}$ is obtained from the one of $A_{\sigma \circ \theta_{i_{1}} \circ \cdots \circ \theta_{i_{r-1}}}$ by tilting with respect to the torsion pair obtained by the simple module.

Combine with the descriptions in Section 1.2, we can see the claim by induction with respect to $r$.

Let $S_{i}^{\zeta}$ be the simple $A_{\sigma}^{\zeta}$-module associated to the vertex $i$. For $\zeta \in C_{\theta}$, we have

$$
\left[S_{i}^{\zeta}\right]=\alpha(\theta, i) \in K_{\text {num }}\left(\bmod _{\text {fin }} A_{\sigma}\right)
$$

under the induced isomorphism

$$
K_{\mathrm{num}}\left(\bmod _{\mathrm{fin}} A_{\sigma}\right) \simeq K_{\mathrm{num}}\left(\bmod _{\mathrm{fin}} A_{\sigma}^{\zeta}\right),
$$

\section{Definition of the invariants}

\subsection{Ideal sheaf associated to Young diagrams}

In this paper, we regard a Young diagram as a subset of $\left(\mathbb{Z}_{>0}\right)^{2}$. For a Young diagram $\lambda$, let $\Lambda^{x}(\lambda)$ (respectively $\Lambda^{y}(\lambda)$ or $\left.\Lambda^{z}(\lambda)\right)$ be the subset of $\left(\mathbb{Z}_{>0}\right)^{3}$ consisting of the elements $(x, y, z) \in\left(\mathbb{Z}_{>0}\right)^{3}$ such that $(y, z) \in \lambda$ (respectively $(z, x) \in \lambda$ or $\left.(x, y) \in \lambda\right)$. Given a triple $\left(\lambda_{x}, \lambda_{y}, \lambda_{z}\right)$ of Young diagrams, let $\Lambda^{\min }=\Lambda_{\lambda_{x}, \lambda_{y}, \lambda_{z}}^{\min }$ be the following subset of $\left(\mathbb{Z}_{>0}\right)^{3}$ :

$$
\Lambda^{\min }:=\Lambda^{x}\left(\lambda_{x}\right) \cup \Lambda^{y}\left(\lambda_{y}\right) \cup \Lambda^{z}\left(\lambda_{z}\right) \subset\left(\mathbb{Z}_{>0}\right)^{3} .
$$

A subset $\Lambda$ of $\left(\mathbb{Z}_{>0}\right)^{3}$ is said to be a 3-dimensional Young diagram of type $\left(\lambda_{x}, \lambda_{y}, \lambda_{z}\right)$ if the following conditions are satisfied:

- if $(x, y, z) \notin \Lambda$, then $(x+1, y, z),(x, y+1, z),(x, y, z+1) \notin \Lambda$,

- $\Lambda \supset \Lambda^{\text {min }}$, and

- $\left|\Lambda \backslash \Lambda^{\min }\right|<\infty$.

For a 3-dimensional Young diagram $\Lambda \subset\left(\mathbb{Z}_{>0}\right)^{3}$, we define an ideal $I_{\Lambda} \subset \mathbb{C}[X, Y, Z]$ by

$$
I_{\Lambda}:=\bigoplus_{(x, y, z) \notin \Lambda} \mathbb{C} \cdot X^{x} Y^{y} Z^{z}
$$

This is invariant with respect to the torus action $T:=\left(\mathbb{C}^{*}\right)^{3} \curvearrowright \mathbb{C}^{3}$. On the other hand, any torus invariant ideal can be described in this way.

Recall that the toric graph of $Y_{\sigma}$ has 
- $L$ vertices,

- $\quad L-1$ closed edges, and

- $L+2$ open edges.

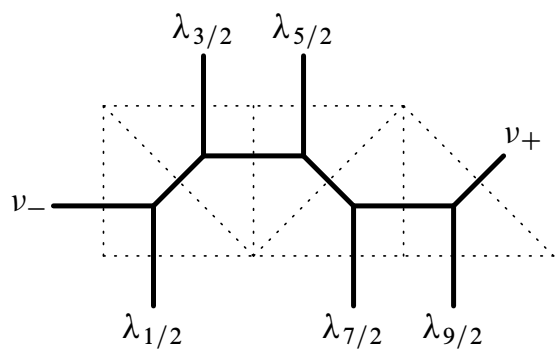

Figure 1: A toric graph and Young diagrams

The set of torus invariant ideal sheaf on $Y$ is parametrized by the following data (see Maulik, Nekrasov, Okounkov and Pandharipande [21]):

- a pair of Young diagrams $\underline{v}=\left(v_{+}, v_{-}\right)$and an $L$-tuple of Young diagrams $\underline{\lambda}=\left(\lambda^{(1 / 2)}, \ldots, \lambda^{(L-1 / 2)}\right)$ corresponding to $L+2$ open edges,

- an $(L-1)$-tuple of Young diagrams $v^{(1)}, \ldots, v^{(L-1)}$ corresponding to closed edges, and

- an $L$-tuple of 3-dimensional Young diagrams $\Lambda^{(1 / 2)}, \ldots, \Lambda^{(L-1 / 2)}$ corresponding to vertices such that $\Lambda^{(j)}$ is

- of type $\left(\lambda^{(j)}, v^{(j+1 / 2)},{ }^{\mathrm{t}} v^{(j-1 / 2)}\right)$ if $\sigma(j)=+$,

- of type $\left(\lambda^{(j)},{ }^{\mathrm{t}} v^{(j-1 / 2)}, v^{(j+1 / 2)}\right)$ if $\sigma(j)=-$,

where we put $v^{(0)}:=v_{-}$and $v^{(L)}:=v_{+}$.

Let

$$
\mathcal{I}\left(\underline{v}, \underline{\lambda} ; v^{(1)}, \ldots, v^{(L-1)}\right)
$$

be the ideal associated with the $L$-tuple $\Lambda^{(1 / 2)}, \ldots, \Lambda^{(L-1 / 2)}$ of 3-dimensional Young diagrams where

- $\Lambda^{(j)}=\Lambda_{\lambda^{(j)}, v^{(j+1 / 2)},{ }^{t} v^{(j-1 / 2)}}$ if $\sigma(j)=+$,

- $\Lambda^{(j)}=\Lambda_{\lambda^{(j)}{ }^{t}{ }^{(j-1 / 2)}{ }^{(j+1 / 2)}}$ if $\sigma(j)=-$.

We set

$$
\mathcal{I}_{\underline{v}, \underline{\lambda}}:=\mathcal{I}(\underline{v}, \underline{\lambda} ; \varnothing, \ldots, \varnothing) .
$$




\subsection{Open non-commutative Donaldson-Thomas invariants}

Assume $\zeta \in C_{\theta}$ for some $\theta \in \Theta$. We put

$$
P_{\underline{\underline{\nu}}, \underline{\lambda}}^{\zeta}:=H_{\mathcal{A}^{\zeta}}^{0}\left(\mathcal{I}_{\underline{\underline{\nu}}, \underline{\lambda}}\right) \text {. }
$$

where $H_{\mathcal{A}^{\zeta}}^{*}(-)$ represents the cohomology with respect to the t-structure corresponding to $\mathcal{A}^{\zeta}$.

Example 2.1 (1) In the case when

$$
\underline{v}=\underline{\varnothing}:=(\varnothing, \varnothing), \quad \underline{\lambda}=\underline{\varnothing}:=(\varnothing, \ldots, \varnothing),
$$

we have

$$
P_{\underline{\varnothing}, \underline{\varnothing}}^{\zeta}=\mathcal{I}_{\underline{\varnothing}, \underline{\varnothing}}=\mathcal{O}_{Y_{\sigma}}
$$

for any $\zeta$.

(2) In the case when $L_{+}=L_{-}=1$ and

$$
\underline{v}=\underline{\varnothing}:=(\varnothing, \varnothing), \quad \underline{\lambda}:=(\varnothing, \square),
$$

we have an exact sequence

$$
0 \rightarrow \mathcal{I}(\underline{\varnothing}, \underline{\lambda} ; \square) \rightarrow \mathcal{I}_{\underline{\varnothing}, \underline{\lambda}} \rightarrow \mathcal{O}_{C}(-1) \rightarrow 0 .
$$

We can see that $\mathcal{I}(\underline{\varnothing}, \underline{\lambda} ; \square)$ does not have $\mathcal{O}_{C}(-n)$ as its quotient for $n>0^{7}$. This means that $\mathcal{I}(\underline{\varnothing}, \underline{\lambda} ; \square)$ is in ${ }^{-1} \operatorname{Per}(Y / X)$. Hence

$$
P_{\underline{v}, \underline{\lambda}}^{\zeta}=\mathcal{I}(\underline{\varnothing}, \underline{\lambda} ; \square) \neq \mathcal{I}_{\underline{\varnothing}, \underline{\lambda}} .
$$

for $\zeta=\left(\zeta_{0}, \zeta_{1}\right)$ such that $\zeta_{0}, \zeta_{1}<0$.

Take $\mathbf{v} \in K_{\text {num }}\left(\mathcal{A}_{\text {fin }}^{\zeta}\right) \simeq \mathbb{Z}^{I}$

Definition 2.2 A $(\sigma, \zeta ; \underline{v}, \underline{\lambda})$-pair of type $\mathbf{v}$ is a pair $(V, s)$ of an element $V \in \mathcal{A}_{\text {fin }}^{\zeta}$ with $[V]=\mathbf{v}$ and a surjection $s: P_{\underline{v}, \underline{\lambda}}^{\zeta} \rightarrow V$ in $\mathcal{A}^{\zeta}$.

Two $(\sigma, \zeta ; \underline{\nu}, \underline{\lambda})$-pairs $(V, s)$ and $\left(V^{\prime}, s^{\prime}\right)$ are said to be equivalent if there exists an isomorphism between $V$ and $V^{\prime}$ compatible with $s$ and $s^{\prime}$.

${ }^{7}$ Suppose that we have a surjection from $\mathcal{I}(\varnothing, \underline{\lambda} ; \square)$ to $\mathcal{O}_{C}(-n)$. We may assume that the map is torus equivariant. Then the kernel is described by a pair of 3-dimensional Young diagrams which is obtained by removing some boxes from the pair of 3 -dimensional Young diagrams associated to $\mathcal{I}(\underline{\varnothing}, \underline{\lambda} ; \square)$. Then we can see that we can not remove boxes so that the cokernel is $\mathcal{O}_{C}(-n)(n>0)$. 
Theorem 2.3 There is a coarse moduli scheme $\mathfrak{M}^{\text {ncDT }}(\zeta, \underline{v}, \underline{\lambda} ; \mathbf{v})$ parameterizing equivalence classes of $(\sigma, \zeta ; \underline{\nu}, \underline{\lambda})$-pairs $(V, s)$ with $[V]=\mathbf{v}$.

The proof of this theorem is given in Section 5.1.

Definition 2.4 We define the generating function of the Euler characteristic version of open non-commutative Donaldson-Thomas invariants

$$
\mathcal{Z}_{\sigma, \zeta ; \underline{\underline{\nu}}, \underline{\lambda}}^{\mathrm{E}-\mathrm{ncDT}}\left(q_{0}, \ldots, q_{L-1}\right):=\sum_{\mathbf{v}} e\left(\mathfrak{M}^{\mathrm{ncDT}}(\zeta, \underline{v}, \underline{\lambda} ; \mathbf{v})\right) \cdot\left(\mathbf{q}_{\theta}\right)^{\mathbf{v}},
$$

where $\left(\mathbf{q}_{\theta}\right)^{\mathbf{v}}:=\prod\left(\mathbf{q}^{\alpha(\theta, i)}\right)^{v_{i}}$. (We use the monomial $\mathbf{q}^{\alpha(\theta, i)}$ since $\alpha(\theta, i)$ is the numerical class of the simple $A_{\sigma}^{\zeta}$-module $S_{i}^{\zeta}$ (see (1-2)).)

\section{Torus fixed points, crystal melting and vertex operators}

\subsection{Crystal melting model and torus fixed points}

Definition 3.1 Let $\mu$ and $\mu^{\prime}$ be two Young diagrams. We say $\mu \stackrel{+}{\succ} \mu^{\prime}$ if the row lengths satisfy

$$
\mu_{1} \geq \mu_{1}^{\prime} \geq \mu_{2} \geq \mu_{2}^{\prime} \geq \cdots,
$$

and $\mu \check{\succ} \mu^{\prime}$ if the column lengths satisfy

$$
{ }^{\mathrm{t}} \mu_{1} \geq{ }^{\mathrm{t}} \mu_{1}^{\prime} \geq{ }^{\mathrm{t}} \mu_{2} \geq{ }^{\mathrm{t}} \mu_{2}^{\prime} \geq \cdots .
$$

Definition 3.2 Let $\Pi$ denote the set of all Young diagrams. A transition $\mathcal{V}$ of Young diagrams of type $(\sigma, \theta ; \underline{v}, \underline{\lambda})$ is a map $\mathcal{V}: \mathbb{Z} \rightarrow \Pi$ such that

- $\mathcal{V}(n)=v_{-}$for $n \ll 0$ and $\mathcal{V}(n)=v_{+}$for $n \gg 0$,

- $\mathcal{V}(h-\underline{\lambda} \circ \theta(h) / 2) \stackrel{\sigma \circ \theta(h)}{\succ} \mathcal{V}(h+\underline{\lambda} \circ \theta(h) / 2)$.

Definition 3.3 For a transition $\mathcal{V}$ of Young diagram of type $(\sigma, \theta ; \underline{v}, \underline{\lambda})$, we put

$$
P(\mathcal{V})_{i}:=\left\{(n, x, y) \in \mathbb{Z} \times\left(\mathbb{Z}_{\geq 0}\right)^{2} \mid n \equiv i(\bmod L),(x, y) \notin \mathcal{V}(n)\right\}
$$

and $P(\mathcal{V}):=\sqcup_{i} P(\mathcal{V})_{i}$. We use the notation $p(n, x, y)$ for an element in $P(\mathcal{V})$.

Lemma 3.4 [26, Section 3.3.3, Remark 3.7] There is a transition $\mathcal{V}_{\min }=\mathcal{V}_{\min }^{\sigma, \theta ; \underline{\nu}, \underline{\lambda}}$ of Young diagrams of type $(\sigma, \theta ; \underline{\nu}, \underline{\lambda})$ such that for any transition $\mathcal{V}$ of Young diagrams of type $(\sigma, \theta ; \underline{\nu}, \underline{\lambda})$ we have $P\left(\mathcal{V}_{\text {min }}\right) \supseteq P(\mathcal{V})$. 
Proof In [26], we use the notation $v$ and $\lambda$ instead of $\underline{v}$ and $\underline{\lambda}$. In [26, Section 3.3.3], a map $G_{\sigma, \lambda, \theta}^{v}$ is given. As is mentioned in [26, Remark 3.7], this map gives a sequence of Young diagrams $\mathcal{V}_{\text {min }}^{\sigma, \theta} \underline{\underline{v}, \underline{\lambda}}$, which satisfies the condition.

Definition 3.5 A crystal of type $(\sigma, \theta ; \underline{v}, \underline{\lambda})$ is a subset $P(\mathcal{V})$ of $P\left(\mathcal{V}_{\min }\right)$ such that $\left|P\left(\mathcal{V}_{\min }\right) \backslash P(\mathcal{V})\right|<\infty$.

The lemma above claims that giving a transition $\mathcal{V}$ of Young diagram of type $(\sigma, \theta ; \underline{\nu}, \underline{\lambda})$ is equivalent to giving a crystal $P(\mathcal{V})$ of type $(\sigma, \theta ; \underline{\nu}, \underline{\lambda})$.

Definition 3.6 Let $M(\mathcal{V})=\oplus_{i} M(\mathcal{V})_{i}$ be the vector space spanned by the elements in $P(\mathcal{V})=\sqcup_{i} P(\mathcal{V})_{i}$. We define an $A_{\sigma \circ \theta}$-action on $M(\mathcal{V})$ by

$$
\begin{aligned}
h_{j}^{+}(p(h-1 / 2, x, y)) & = \begin{cases}p(h+1 / 2, x, y) & (\underline{\lambda} \circ \theta(h)=+), \\
p(h+1 / 2, x+1, y) & (\underline{\lambda} \circ \theta(h)=-, \sigma \circ \theta(h)=-), \\
p(h+1 / 2, x, y+1) & (\underline{\lambda} \circ \theta(h)=-, \sigma \circ \theta(h)=+),\end{cases} \\
h_{j}^{-}(p(h+1 / 2, x, y)) & = \begin{cases}p(h-1 / 2, x, y) & (\underline{\lambda} \circ \theta(h)=-), \\
p(h-1 / 2, x+1, y) & (\underline{\lambda} \circ \theta(h)=+, \sigma \circ \theta(h)=-), \\
p(h-1 / 2, x, y+1) & (\underline{\lambda} \circ \theta(h)=+, \sigma \circ \theta(h)=+),\end{cases} \\
r_{i}(p(n, x, y)) & = \begin{cases}p(n, x+1, y) & (\sigma \circ \theta(n-1 / 2)=\sigma \circ \theta(n+1 / 2)=+), \\
p(n, x, y+1) & (\sigma \circ \theta(n-1 / 2)=\sigma \circ \theta(n+1 / 2)=-),\end{cases}
\end{aligned}
$$

Proposition 3.7 For $\zeta \in C_{\theta}$, we have $M\left(\mathcal{V}_{\min }^{\sigma, \theta ; \underline{v}, \underline{\lambda}}\right) \simeq P_{\underline{\underline{v}}, \underline{\lambda}}^{\zeta}$ as an $A_{\sigma \circ \theta}$-module.

The proof of this proposition is given in Section 6.3.

Remark 3.8 The $A_{\sigma \circ \theta}$-module $M\left(\mathcal{V}_{\min }^{\sigma, \theta ; \underline{v}, \underline{\lambda}}\right)$ coincides with $M_{\sigma, \lambda, \nu, \theta}^{\max }$ defined in [26, Section 3.3.3].

Proposition 3.9 Let $\left(P_{\underline{v}, \underline{\lambda}}^{\zeta} \rightarrow V\right) \in \mathfrak{M}(\underline{\nu}, \underline{\lambda}, \zeta ; \mathbf{v})$ be a torus fixed point. Then the kernel of the map is described as $M(\mathcal{V})$ for a transition $\mathcal{V}$.

Proof Take a one parameter subgroup $\rho: T \rightarrow \prod \operatorname{GL}\left(\left(P_{\underline{v}, \underline{\lambda}}^{\zeta}\right)_{i}\right)$ such that $\rho(t) * P_{\underline{v}, \underline{\lambda}}^{\zeta}=$ $t \cdot P_{\underline{v}, \underline{\lambda}}^{\zeta}$. Each element in $P\left(\mathcal{V}_{\min }\right)$ gives an eigenvector for $\rho$ and the eigenvalues are distinct from each other. Hence the kernel is spanned by a subset of $P\left(\mathcal{V}_{\min }\right)$. We can verify that a subset of $P\left(\mathcal{V}_{\min }\right)$ gives an $A_{\sigma}^{\zeta}$-submodule of $M\left(\mathcal{V}_{\min }\right)$ if and only if it is a crystal. 
Definition 3.10 For a transition $\mathcal{V}$ of Young diagram of type $(\sigma, \theta ; \underline{v}, \underline{\lambda})$, we define the weight $\mathbf{v}(\mathcal{V}) \in \mathbb{Z}^{I}$ of $\mathcal{V}$ by

$$
\mathbf{v}(\mathcal{V})_{i}:=\sharp\left\{p(n, x, y) \in P\left(\mathcal{V}_{\min }\right) \backslash P(\mathcal{V}) \mid n \equiv i(\bmod L)\right\} .
$$

\section{Definition 3.11}

$$
\mathcal{Z}_{\sigma, \theta ; \underline{v}, \underline{\lambda}}^{\text {crystal }}\left(q_{0}, \ldots, q_{L-1}\right):=\sum_{\mathcal{V}: \text { of type }(\sigma, \theta ; \underline{\nu}, \underline{\lambda})}\left(\mathbf{q}_{\theta}\right)^{\mathbf{v}(\mathcal{V})} .
$$

Corollary 3.12

$$
\mathcal{Z}_{\sigma, \zeta ; \underline{\underline{v}, \underline{\lambda}}}^{\mathrm{E}-\mathrm{ncDT}}\left(q_{0}, \ldots, q_{L-1}\right)=\mathcal{Z}_{\sigma, \theta ; \underline{v}, \underline{\lambda}}^{\mathrm{crystal}}\left(q_{0}, \ldots, q_{L-1}\right) .
$$

\subsection{Crystal melting and vertex operators}

Let $\mathbb{K}:=\mathbb{C}\left(\left(q_{i}\right)\right)$ be the ring of Laurent formal power series with variables $q_{i}(i \in I)$ and $\Pi$ be the set of Young diagrams. We define the Fock space $\left(\wedge^{\frac{\infty}{2}}\right)_{0}$ by

$$
\left(\wedge^{\frac{\infty}{2}}\right)_{0}:=\bigoplus_{\mu \in \Pi} \mathbb{K} \cdot \mu
$$

Let $\langle-\mid-\rangle$ be the $\mathbb{K}$-bilinear inner product under which $\{\mu\}$ are orthonormal.

We will use the "bra-ket" notation:

$$
\left\langle\mu^{\prime}|A| \mu\right\rangle:=\left\langle\mu^{\prime} \mid A \mu\right\rangle=\left\langle{ }^{\mathrm{t}} A \mu^{\prime} \mid \mu\right\rangle,
$$

where $A$ is an endomorphism of $\left(\wedge^{\frac{\infty}{2}}\right)_{0}$.

Definition 3.13 For $p \in \mathbb{K}$, we define the vertex operators $\Gamma_{ \pm}^{ \pm}(p):\left(\wedge^{\frac{\infty}{2}}\right)_{0} \rightarrow\left(\wedge^{\frac{\infty}{2}}\right)_{0}$ by

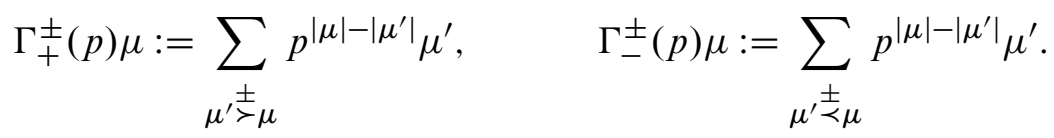

Lemma 3.14 (see Young [12, Lemma 3.3]) For $p, p^{\prime} \in \mathbb{K}$, we have

$$
\left[\Gamma_{\iota_{1}}^{\varepsilon_{1}}\left(p_{1}\right), \Gamma_{\iota_{2}}^{\varepsilon_{2}}\left(p_{2}\right)\right]=\left(1-\varepsilon_{1} \varepsilon_{2} p_{1}^{\iota_{1}} p_{2}^{\iota_{2}}\right)^{-\iota_{1} \varepsilon_{1} \varepsilon_{2} \delta_{\iota_{1}+\iota_{2}}} .
$$

For $w^{\prime} \in \mathbb{Z}^{I}$, we set

$$
\left(\mathbb{Z}^{I}\right)_{\leq w^{\prime}}:=\left\{w \in \mathbb{Z}^{I} \mid w_{i} \leq w_{i}^{\prime} \text { for all } i \in I\right\} .
$$


Lemma 3.15 (see Young [12]) Let $J$ be a countable set and $w: J \rightarrow \mathbb{Z}^{I}$ be a map such that $w^{-1}\left(\left(\mathbb{Z}^{I}\right)_{\leq w^{\prime}}\right)$ is finite for any $w^{\prime} \in \mathbb{Z}^{I}$. Put $p_{j}:=\mathbf{q}^{w(j)}$. Note that for any map $\varepsilon: J \rightarrow\{ \pm\}$ the operator $\prod_{j \in J} \Gamma_{ \pm}^{\varepsilon(j)}\left(p_{j}\right)$ is well-defined. Then we have

$$
\begin{aligned}
\left\langle\mu^{\prime}\left|\prod \Gamma_{-}^{+}\left(p_{i}\right)\right| \mu\right\rangle & =\left\langle\mu\left|\prod \Gamma_{+}^{+}\left(p_{i}\right)\right| \mu^{\prime}\right\rangle=s_{\left(\mu^{\prime} \backslash \mu\right)}\left(p_{i}\right), \\
\left\langle\mu^{\prime}\left|\prod \Gamma_{-}^{-}\left(p_{i}\right)\right| \mu\right\rangle & =\left\langle\mu\left|\prod \Gamma_{+}^{-}\left(p_{i}\right)\right| \mu^{\prime}\right\rangle=s_{\left({ }^{\prime} \mu^{\prime} \backslash^{\mathrm{t}} \mu\right)}\left(p_{i}\right)
\end{aligned}
$$

where $s_{\left(\mu^{\prime} \backslash \mu\right)}$ and $s_{\left({ }^{t} \mu^{\prime} \backslash{ }^{\prime} \mu\right)}$ denote the skew Schur functions.

Let $f(h)\left(h \in \mathbb{Z}_{\mathrm{h}}\right)$ be a sequence of operators. If the composition of the operator

$$
\cdots \circ f\left(\theta^{-1}(h-1)\right) \circ f\left(\theta^{-1}(h)\right) \circ f\left(\theta^{-1}(h+1)\right) \circ \cdots
$$

is well-defined, we denote this by

$$
\prod_{h \in \mathbb{Z}_{\mathrm{h}}}^{\theta} f(h) .
$$

We set $\alpha_{h}:=\alpha_{[1 / 2, h]}$ and $q_{h}:=\mathbf{q}^{\alpha_{h}}$ and define the monomial

$$
\mathbf{q}(\sigma, \theta ; \underline{\nu}, \underline{\lambda}):=\prod_{h \in \mathbb{Z}_{\mathrm{h}}}\left(q_{h}\right)^{\left|\mathcal{V}_{\min }(h-1 / 2)\right|-\left|\mathcal{V}_{\min }(h+1 / 2)\right|} .
$$

The following claim is clear from the definitions:

\section{Proposition 3.16}

$$
\mathcal{Z}_{\sigma, \theta ; \underline{\underline{v}}, \underline{\lambda}}^{\mathrm{crystal}}\left(q_{0}, \ldots, q_{L-1}\right)=\left\langle v_{-}\left|\prod_{h \in \mathbb{Z}_{\mathrm{h}}}^{\theta} \Gamma_{\lambda(h)}^{\sigma(h)}\left(q_{h}\right)\right| v_{+}\right\rangle \cdot \mathbf{q}(\sigma, \theta ; \underline{v}, \underline{\lambda}) .
$$

\subsection{Computation of the generating function}

For a symmetric function $f=f\left(p_{1}, p_{2}, \ldots\right)$, let $f^{*}$ be the symmetric function given by $f^{*}\left(p_{1}, p_{2}, \ldots\right)=f\left(p_{1}^{-1}, p_{2}^{-1}, \ldots\right)$. For a subset $S \subset \mathbb{Z}_{\mathrm{h}}$, let $f(S ; \mathbf{q})$ denote the symmetric function given by substituting $\left\{q_{h} \mid h \in S\right\}$ for $f$. We set

$$
S_{\underline{\lambda}}^{\iota}:=\left\{h \in \mathbb{Z}_{\mathrm{h}} \mid \underline{\lambda}(h)=\iota\right\}, \quad S_{\sigma, \underline{\lambda}}^{\varepsilon, \iota}:=\left\{h \in \mathbb{Z}_{\mathrm{h}} \mid \sigma(h)=\varepsilon, \underline{\lambda}(h)=\iota\right\} .
$$

The following lemma is a direct consequence of Lemma 3.14: 


\section{Lemma 3.17}

$$
\begin{aligned}
\left\langle v_{-}\right| & \prod_{h \in S_{\underline{\lambda}}^{+}} \Gamma_{\lambda(h)}^{+}\left(q_{h}\right) \cdot \prod_{h \in S_{\underline{\lambda}}^{-}} \Gamma_{\lambda(h)}^{-}\left(q_{h}\right)\left|v_{+}\right\rangle= \\
& \sum_{\nu_{-} \supseteq v_{1} \supseteq v_{2} \subseteq v_{3} \subseteq v_{+}} s_{\nu_{-} \backslash v_{1}}\left(S_{\sigma, \underline{\lambda}}^{+,+} ; \mathbf{q}\right) \cdot{ }^{\mathrm{t}} s_{v_{1} \backslash v_{2}}^{*}\left(S_{\sigma, \underline{\lambda}}^{-,+} ; \mathbf{q}\right) \cdot s_{\nu_{3} \backslash v_{2}}\left(S_{\sigma, \underline{\lambda}}^{+,--} ; \mathbf{q}\right) \cdot{ }^{\mathrm{t}} s_{v_{+} \backslash v_{3}}^{*}\left(S_{\sigma, \underline{\lambda}}^{-,-} ; \mathbf{q}\right)
\end{aligned}
$$

For $\alpha=\alpha_{\left[h, h^{\prime}\right]} \in \Delta$, we set $\sigma(\alpha)=-\sigma(h) \sigma\left(h^{\prime}\right)$ and

$$
\alpha_{0}:=\sharp\left\{m \in \mathbb{Z} \mid h<m L<h^{\prime}\right\} .
$$

We can easily verify the following:

Lemma 3.18 For $\alpha \in \Delta^{\text {re }}$ and for any $\underline{\lambda}$, we have

$$
\sharp\left\{\left(h, h^{\prime}\right) \in\left(\mathbb{Z}_{\mathrm{h}}\right)^{2} \mid \alpha_{\left[h, h^{\prime}\right]}=\alpha, \underline{\lambda}(h)=-, \underline{\lambda}\left(h^{\prime}\right)=+\right\}=\alpha_{0} .
$$

Let

$$
M(1, t):=\prod_{m>0}\left(1-t^{m}\right)^{-m}
$$

be MacMahon function and

$$
s_{\lambda}\left(t^{-\rho}\right):=s_{\lambda}\left(t^{1 / 2}, t^{3 / 2}, \ldots\right)
$$

be the specialization of Schur function. The next equation follows from the hook length formula:

\section{Lemma 3.19}

$$
\prod\left(1-t^{m^{\prime}-m}\right)=M(1, t) \cdot s_{\lambda(j)}\left(t^{-\rho}\right)
$$

where the product in the left hand side is taken over the set

$$
\left\{\left(m, m^{\prime}\right) \mid m<m^{\prime}, \underline{\lambda}(m L+j)=-, \underline{\lambda}\left(m^{\prime} L+j\right)=+\right\} .
$$

\section{Definition 3.20}

$$
\begin{aligned}
& \mathcal{Z}_{\sigma, \underline{\nu}, \underline{\lambda}}^{\zeta>0}\left(q_{0}, \ldots, q_{L-1}\right):=\mathbf{q}(\sigma, \theta ; \underline{v}, \underline{\lambda}) . \\
& \left(\sum_{\nu_{-} \supseteq v_{1} \supseteq v_{2} \subseteq v_{3} \subseteq v_{+}} s_{\nu_{-} \backslash v_{1}}\left(S_{\sigma, \underline{\lambda}}^{+,-} ; \mathbf{q}\right) \cdot{ }^{\mathrm{t}} s_{v_{1} \backslash \nu_{2}}^{*}\left(S_{\sigma, \underline{\lambda}}^{-,-} ; \mathbf{q}\right) \cdot s_{\nu_{3} \backslash v_{2}}\left(S_{\sigma, \underline{\lambda}}^{+,+} ; \mathbf{q}\right) \cdot{ }^{\mathrm{t}} s_{v_{+} \backslash v_{3}}^{*}\left(S_{\sigma, \underline{\lambda}}^{-,+} ; \mathbf{q}\right)\right) .
\end{aligned}
$$

Combining Corollary 3.12, Proposition 3.16, and Lemmas 3.14, 3.17 and 3.18, we get the following explicit formula: 
Theorem 3.21 If we put $t=q_{0} \cdots \cdots q_{L-1}$, then we have $\mathcal{Z}_{\sigma, \zeta ; \underline{v}, \underline{\lambda}}^{\mathrm{E}-\mathrm{ncDT}}\left(q_{0}, \ldots, q_{L-1}\right)=$ $M(1, t)^{L} \cdot \prod_{j} s_{\lambda(j)}\left(t^{-\rho}\right) \cdot\left(\prod_{\alpha \in \Delta^{\mathrm{re},}+, \theta(\alpha)<0,}\left(1+\sigma(\alpha) \mathbf{q}^{\alpha}\right)^{\sigma(\alpha) \alpha_{0}}\right) \cdot \mathcal{Z}_{\sigma, \underline{v}, \underline{\lambda}}^{\zeta>0}\left(q_{0}, \ldots, q_{L-1}\right)$.

Corollary 3.22 The normalized generating function

$$
\mathbf{q}(\sigma, \theta ; \underline{\nu}, \underline{\lambda}) \cdot \mathcal{Z}_{\sigma, \theta ; \underline{\nu}, \underline{\lambda}}^{\mathrm{E}-\mathrm{ncDT}}\left(q_{0}, \ldots, q_{L-1}\right) / \mathcal{Z}_{\sigma, \theta, \underline{\varnothing}, \underline{\varnothing}}^{\mathrm{E}-\mathrm{ncDT}}\left(q_{0}, \ldots, q_{L-1}\right)
$$

does not depend on $\theta$.

\section{Open Donaldson-Thomas invariants and topological ver- tex}

\subsection{Open Donaldson-Thomas invariants}

Take $\beta \in H_{2}\left(Y_{\sigma}, \mathbb{Z}\right)$ and $n \in \mathbb{Z}$. Note that $H_{2}\left(Y_{\sigma}, \mathbb{Z}\right)$ has the natural basis

$$
\left\{\left[C_{i}\right]\right\}_{i=1, \ldots, L-1},
$$

where $C_{i} \simeq \mathbb{P}^{1}$ is an irreducible component of the fiber $f^{-1}(0)$ of the contraction $f: Y_{\sigma} \rightarrow X$. The derived equivalence induces the following isomorphism:

$$
\begin{aligned}
\psi: K_{\text {num }}\left(\bmod _{\mathrm{fin}} A_{\sigma}\right) & \stackrel{\sim}{\longrightarrow} H_{2}\left(Y_{\sigma}, \mathbb{Z}\right) \oplus \mathbb{Z}, \\
{\left[S_{i}\right](i \neq 0) } & \longmapsto\left[C_{i}\right] \in H_{2}\left(Y_{\sigma}, \mathbb{Z}\right), \\
{\left[S_{0}\right]+\cdots+\left[S_{L-1}\right] } & \longmapsto 1 \in \mathbb{Z} .
\end{aligned}
$$

Definition 4.1 A $(\underline{v}, \underline{\lambda})$-pair of type $(\beta, n)$ is a pair $(F, s)$ of a coherent sheaf $F \in$ $\operatorname{Coh}_{\mathrm{cpt}}\left(Y_{\sigma}\right)$ and a morphism $s: \mathcal{I}_{\underline{v}, \underline{\lambda}} \rightarrow F$ such that $c_{2}(F)=\beta, \chi(F)=n$ and $s$ is surjective.

Two $(\underline{v}, \underline{\lambda})$-pairs $(F, s)$ and $\left(F^{\prime}, s^{\prime}\right)$ are said to be equivalent if there exists an isomorphism between $F$ and $F^{\prime}$ compatible with $s$ and $s^{\prime}$.

Recall that in [24] we construct a tilting vector bundle $\mathcal{P}:=\mathcal{O}_{Y_{\sigma}} \oplus \bigoplus_{i} L_{i}$ on $Y_{\sigma}$ following van den Bergh [6]. In particular, we have

$$
(2-L)\left[\mathcal{O}_{Y_{\sigma}}\right]+\sum_{i}\left[L_{i}\right]=[\mathcal{L}] \in K_{0}\left(\operatorname{Coh}\left(Y_{\sigma}\right)\right)
$$

where $\mathcal{L}$ is an ample line bundle on $Y_{\sigma}$. 
The functor $\mathbb{R} \operatorname{Hom}(\mathcal{L},-)$ gives an equivalence between $D^{b} \operatorname{Coh}\left(Y_{\sigma}\right)$ (respectively $\left.D_{\mathrm{cpt}}^{b} \operatorname{Coh}\left(Y_{\sigma}\right)\right)$ and $D^{b}\left(\bmod A_{\sigma}\right)\left(\right.$ respectively $\left.D_{\text {fin }}^{b}\left(\bmod A_{\sigma}\right)\right)$, which restricts to an equivalence between ${ }^{-1} \operatorname{Per}\left(Y_{\sigma} / X\right)$ and $\bmod A_{\sigma}$. Here ${ }^{-1} \operatorname{Per}\left(Y_{\sigma} / X\right)$ is the full subcategory of $D^{b} \operatorname{Coh}\left(Y_{\sigma}\right)$ consisting of elements $E$ satisfying the following conditions:

- $H_{\mathrm{Coh}\left(Y_{\sigma}\right)}^{i}(E)=0$ unless $i=0,-1$,

- $\mathbb{R}^{1} f_{*}\left(H_{\mathrm{Coh}\left(Y_{\sigma}\right)}^{0}(E)\right)=0$ and $\mathbb{R}^{0} f_{*}\left(H_{\mathrm{Coh}\left(Y_{\sigma}\right)}^{-1}(E)\right)=0$,

- $\operatorname{Hom}\left(H_{\mathrm{Coh}\left(Y_{\sigma}\right)}^{0}(E), C\right)=0$ for any sheaf $C$ on $Y$ satisfying $\mathbb{R} f_{*}(C)=0$.

Take $\zeta_{\text {cyc }}^{\circ}=(1-L, 1,1, \ldots, 1)$. Note that $\left(\zeta^{\circ}, T^{\prime}\right)$ is not on an intersection of two walls for any $T^{\prime} \in \mathbb{R}$ (see Section 1.5).

Lemma 4.2 For an element $E \in \operatorname{Coh}_{\text {cpt }}\left(Y_{\sigma}\right) \cap \bmod _{\text {fin }} A_{\sigma}$ we have

$$
\phi_{Z_{\text {cyc }}}(E) \leq 1 / 2
$$

and for an element $E[1] \in \operatorname{Coh}_{\mathrm{cpt}}\left(Y_{\sigma}\right)[1] \cap \bmod _{\mathrm{fin}} A_{\sigma}$ we have

$$
\phi_{Z_{\text {cyc }}}(E[1])>1 / 2 .
$$

Proof By (4-1), for an element $E \in \mathrm{Coh}_{\mathrm{cpt}}\left(Y_{\sigma}\right)$ we have

$$
\zeta_{\text {cyc }}^{\circ} \cdot \underline{\operatorname{dim}} E=h^{0}\left(E, Y_{\sigma}\right)-h^{0}\left(E \otimes \mathcal{L}, Y_{\sigma}\right) \leq 0 .
$$

and the equality holds if and only if $E$ is 0 -dimensional. Since any 0 -dimensional sheaf is in $\bmod _{\text {fin }} A_{\sigma}$, the claim follows.

\section{Lemma 4.3}

$$
\mathcal{D}_{\text {fin }}^{\zeta_{\text {cyc }}^{\circ}}[1 / 2,0) \subset \operatorname{Coh}_{\text {cpt }}\left(Y_{\sigma}\right), \quad \mathcal{D}_{\text {fin }}^{\zeta_{\text {cyc }}^{\circ}}[1,1 / 2) \subset \operatorname{Coh}_{\text {cpt }}\left(Y_{\sigma}\right)[1] .
$$

Proof Let $E \in D_{\text {fin }}^{b}\left(\bmod A_{\sigma}\right)$ be a $Z_{\zeta_{\text {cyc }}^{\circ}}$-semistable object with $1 / 2 \geq \phi_{Z_{\zeta_{\text {cyc }}^{\circ}}}(E)>0$. By the canonical exact sequence

$$
0 \rightarrow H_{\mathrm{Coh}\left(Y_{\sigma}\right)}^{-1}(E)[1] \rightarrow E \rightarrow H_{\mathrm{Coh}\left(Y_{\sigma}\right)}^{0}(E) \rightarrow 0 .
$$

Since $H_{\mathrm{Coh}\left(Y_{\sigma}\right)}^{-1}(E)[1] \in \operatorname{Coh}_{\mathrm{cpt}}\left(Y_{\sigma}\right)[1] \cap \bmod _{\mathrm{fin}} A_{\sigma}$, we have

$$
\phi_{Z_{\text {cyc }}}\left(H_{\operatorname{Coh}\left(Y_{\sigma}\right)}^{-1}(E)[1]\right)>1 / 2
$$

by Lemma 4.2. Then we can see $H_{\mathrm{Coh}\left(Y_{\sigma}\right)}^{-1}(E)[1]=0$ and so $E \in \operatorname{Coh}_{\mathrm{cpt}}\left(Y_{\sigma}\right)$. We can show the right inclusion in the same way. 


\section{Proposition 4.4}

$$
\mathcal{D}_{\text {fin }}^{\zeta_{\text {cyc }}^{\circ}}[1 / 2,-1 / 2) \simeq \operatorname{Coh}_{\text {cpt }}\left(Y_{\sigma}\right)
$$

Proof Every object $F \in \mathcal{D}_{\text {fin }}^{\zeta_{\text {cyc }}^{\circ}}[1 / 2,-1 / 2)$ fits into a short exact sequence

$$
0 \rightarrow E \rightarrow F \rightarrow G \rightarrow 0
$$

for some pair of objects $E \in \mathcal{D}_{\text {fin }}^{\zeta_{\text {cyc }}^{\circ}}[1 / 2,0)$ and $G \in \mathcal{D}_{\text {fin }}^{\zeta_{\text {cyc }}^{\circ}}\left[0,-1 / 2 \xi_{\xi}\right.$. By Lemma 4.3, we have $E, G \in \operatorname{Coh}_{\text {cpt }}\left(Y_{\sigma}\right)$ and so $F \in \operatorname{Coh}_{\text {cpt }}\left(Y_{\sigma}\right)$. Since both $\mathcal{D}_{\text {fin }}^{\zeta_{\text {cyc }}}[1 / 2,-1 / 2)$ and $\mathrm{Coh}_{\mathrm{cpt}}\left(Y_{\sigma}\right)$ are cores of t-structures, the inclusion is equivalence and the claim follows.

Lemma 4.5 Let $G$ be an $A_{\sigma}$-module. Suppose that $\operatorname{Hom}(X, G)=0$ for any finite dimensional $A_{\sigma}$-module $X$. Then we have $G \in \operatorname{Coh}\left(Y_{\sigma}\right)$.

Proof Recall that $\bmod \left(A_{\sigma}\right)$ coincides with ${ }^{-1} \operatorname{Per}(Y / X)$ (Theorem 1.2). Thus we have the following exact sequence in $\bmod \left(A_{\sigma}\right)$ :

$$
0 \rightarrow H_{\operatorname{coh}\left(Y_{\sigma}\right)}^{-1}(G) \rightarrow G \rightarrow H_{\mathrm{coh}\left(Y_{\sigma}\right)}^{0}(G) \rightarrow 0 .
$$

Since the restriction of an element in ${ }^{-1} \operatorname{Per}(Y / X)$ to the smooth locus of $X$ is a sheaf, the support of $H_{\operatorname{coh}\left(Y_{\sigma}\right)}^{-1}(G)$ is compact. Thus, as an $A_{\sigma}$-module, $H_{\operatorname{coh}\left(Y_{\sigma}\right)}^{-1}(G)$ is finite dimensional.

By the assumption we have $H_{\operatorname{coh}\left(Y_{\sigma}\right)}^{-1}(G)=0$. Hence the claim follows.

\section{Lemma 4.6}

$$
\mathcal{D}^{\zeta_{\text {cyc }}^{\circ}}[1 / 2,0)^{\perp} \subset \operatorname{Coh}\left(Y_{\sigma}\right)
$$

Proof In the proof of Proposition 1.5, we show that any element $F \in \mathcal{D}_{\text {cyc }}^{\zeta^{\circ}}[1 / 2,0)^{\perp}$ fits into an exact sequence

$$
0 \rightarrow E \rightarrow F \rightarrow G \rightarrow 0
$$

where $E \in \mathcal{D}_{\text {fin }}^{\zeta_{\text {cyc }}^{\circ}}[1 / 2,0)$ and $\operatorname{Hom}(X, G)=0$ for any finite dimensional $A_{\sigma}$-module $X$. By Lemma 4.3 and Lemma 4.5, we have $F \in \operatorname{Coh}\left(Y_{\sigma}\right)$.

\section{Proposition 4.7}

$$
\mathcal{D}_{\text {cyc }}^{\circ}[1 / 2,-1 / 2) \simeq \operatorname{Coh}\left(Y_{\sigma}\right)
$$

Proof Using the previous lemma, we can prove the claim in the same way as Proposition 4.4. 
Theorem 4.8 There is a coarse moduli scheme $\mathfrak{M}^{\mathrm{DT}}(\underline{v}, \underline{\lambda} ; \beta, n)$ parameterizing equivalence classes of $(\underline{v}, \underline{\lambda})$-pairs $(F, s)$ of type $(\beta, n)$.

Proof By the Noetherian property, we can take sufficiently small $T>0$ such that $\mathcal{I}_{\underline{\nu}, \underline{\lambda}} \in \mathcal{A}_{\text {fin }}^{\zeta}$ for $\zeta=\zeta_{\text {cyc }}^{\circ}+T \eta$. Moreover we can assume that for any positive root $\alpha \leq \psi^{-1}(\beta, n)$ and for any $T>T^{\prime}>0, \zeta^{\prime}:=\zeta_{\text {cyc }}^{\circ}+T \eta$ is not on the wall $W_{\alpha}$. Then, using Propositions 4.4 and 4.7 and by the same argument as in [27, Section 2], we can verify that giving a $(\underline{\nu}, \underline{\lambda})$-pair is equivalent to giving a $(\sigma, \zeta ; \underline{v}, \underline{\lambda})$-pair. Hence the claim follows from Theorem 2.3.

Remark 4.9 An alternative construction for $\mathfrak{M}^{\mathrm{DT}}(\underline{\nu}, \underline{\lambda} ; \beta, n)$ is the following: first, take a compactification $\bar{Y}$ of $Y$ and let $\overline{\mathcal{I}_{\underline{v}}, \underline{\lambda}}$ be the ideal sheaf on $\bar{Y}$. Then we can get the moduli scheme as an open subscheme of the quot scheme for $\overline{\mathcal{I}_{\underline{v}}, \underline{\lambda}}$.

Corollary 4.10 Take sufficiently small $T>0$ and put $\zeta=\zeta_{\text {cyc }}^{\circ}+T \eta$, then we have

$$
\mathfrak{M}^{\mathrm{DT}}(\underline{\nu}, \underline{\lambda} ; \beta, n) \simeq \mathfrak{M}^{\mathrm{ncDT}}(\zeta, \underline{v}, \underline{\lambda} ; \mathbf{v}) .
$$

Definition 4.11 We define the generating function

$$
\mathcal{Z}_{\sigma, \underline{\nu}, \underline{\lambda}}^{\mathrm{E}-\mathrm{DT}}\left(q_{1}, \ldots, q_{L-1}, t\right):=\sum_{n, \beta} e\left(\mathfrak{M}^{\mathrm{DT}}(\underline{\nu}, \underline{\lambda} ; \beta, n)\right) \cdot \mathbf{q}^{\beta} t^{n},
$$

where $\mathbf{q}^{\beta}:=\prod\left(q_{i}\right)^{\beta_{i}}$ for $\beta=\sum \beta_{i}\left[C_{i}\right]$.

\subsection{Topological vertex via vertex operators}

Let $\vec{v}=\left(v^{(1)}, \ldots, v^{(L-1)}\right)$ be an $(L-1)$-tuple of Young diagrams and let $\vec{\Lambda}=$ $\left(\Lambda^{(1 / 2)}, \ldots, \Lambda^{(L-1 / 2)}\right)$ be an $L$-tuple of 3 -dimensional Young diagrams such that $\Lambda^{(j)}$ is

- of type $\left(\lambda^{(j)}, v^{(j+1 / 2)},{ }^{\mathrm{t}} v^{(j-1 / 2)}\right)$ if $\sigma(j)=+$,

- of type $\left(\lambda^{(j)},{ }^{\mathrm{t}} v^{(j-1 / 2)}, v^{(j+1 / 2)}\right)$ if $\sigma(j)=-$,

where we put $v^{(0)}:=v_{-}$and $v^{(L)}:=v_{+}$.

For a $(L-1)$-tuple of Young diagrams $\vec{v}$, we define the weight

$$
w(\vec{v}):=\prod_{i} \prod_{(x, y) \in \mathcal{v}^{(i)}} \begin{cases}q_{i} \cdot t^{2 x+1} & \sigma(i+1 / 2)=\sigma(i-1 / 2)=+, \\ q_{i} \cdot t^{2 y+1} & \sigma(i+1 / 2)=\sigma(i-1 / 2)=-, \\ q_{i} \cdot t^{x+y+1} & \sigma(i+1 / 2) \neq \sigma(i-1 / 2),\end{cases}
$$


and for a 3-dimensional Young diagram $\Lambda$ of type $\left(\lambda_{x}, \lambda_{y}, \lambda_{z}\right)$ we define the weight $w(\Lambda)$ by

$$
w(\Lambda):=t^{\left|\Lambda \backslash \Lambda_{\min }\right|} .
$$

For a datum $(\vec{\mu}, \vec{\Lambda})$ as above, we define the weight $w(\vec{\mu}, \vec{\Lambda})$ by

$$
w(\vec{\mu}, \vec{\Lambda}):=w(\vec{v}) \cdot \prod w\left(\Lambda^{(j)}\right) .
$$

The $T:=\left(\mathbb{C}^{*}\right)^{3}$-action on $Y_{\sigma}$ induces a $T$-action on $\mathfrak{M}^{\mathrm{DT}}(\underline{v}, \underline{\lambda} ; \beta, n)$. The following claim is given by Maulik, Nekrasov, Okounkov and Pandharipande [21].

Proposition 4.12 The set $\mathfrak{M}^{\mathrm{DT}}(\underline{\underline{\nu}}, \underline{\lambda} ; \beta, n)^{T}$ of $T$-fixed points is isolated and parametrized by the data $(\vec{\mu}, \vec{\Lambda})$ as above with weight $\mathbf{q}^{\beta} \cdot t^{n}$.

Definition 4.13 We define the generating function

$$
\mathcal{Z}_{\underline{v}, \underline{\lambda}}^{\mathrm{TV}}\left(q_{1}, \ldots, q_{L-1}, t\right):=\sum_{(\vec{\mu}, \vec{\Lambda})} w(\vec{\mu}, \vec{\Lambda}) .
$$

Corollary 4.14 If we put $t=q_{0} \cdots \cdots q_{L-1}$, then we have

$$
\mathcal{Z}_{\sigma, \underline{v}, \underline{\lambda}}^{\mathrm{E}-\mathrm{DT}}\left(q_{0}, \ldots, q_{L-1}\right)=\mathcal{Z}_{\sigma, \underline{v}, \underline{\lambda}}^{\mathrm{TV}}\left(q_{1}, \ldots, q_{L-1}, t\right)
$$

We set

$$
H_{ \pm}^{j}:=\left\{h \in \mathbb{Z}_{\mathrm{h}} \mid \pi(h)=j, \underline{\lambda}(h)= \pm\right\} .
$$

The following claim directly follows from the argument in the paper by Okounkov, Reshetikhin and Vafa [29]:

\section{Proposition 4.15}

$$
\begin{aligned}
& \left.\mathcal{Z}_{\sigma, \theta ; \underline{\underline{\nu}}, \underline{\lambda}}^{\mathrm{TV}}\left(q_{1}, \ldots, q_{L-1}, t\right)\right|_{t=\mathbf{q}^{\delta}}= \\
& \mathbf{q}(\sigma, \theta ; \underline{\nu}, \underline{\lambda}) \cdot\left\langle v_{-}\right| \prod_{h \in H_{\underline{1}} / 2} \Gamma_{-}^{\sigma(1 / 2)}\left(q_{h}\right) \cdot \prod_{h \in H_{+}^{1 / 2}} \Gamma_{+}^{\sigma(1 / 2)}\left(q_{h}\right) \cdot \prod_{h \in H_{\underline{3}} / 2} \Gamma_{-}^{\sigma(3 / 2)}\left(q_{h}\right) . \\
& \ldots \prod_{h \in H_{-}-1 / 2} \Gamma_{-}^{\sigma(L-1 / 2)}\left(q_{h}\right) \cdot \prod_{h \in H_{-}-1 / 2} \Gamma_{+}^{\sigma(L-1 / 2)}\left(q_{h}\right)\left|v_{+}\right\rangle .
\end{aligned}
$$

We set

$$
\Delta_{-}^{\mathrm{re},+}:=\left\{\alpha_{\left[h, h^{\prime}\right]} \in \Delta^{\mathrm{re},+} \mid \pi(h)>\pi\left(h^{\prime}\right)\right\} .
$$

We can compute the generating function in the same way as Theorem 3.21. 


\section{Theorem 4.16}

$\mathcal{Z}_{\sigma, \theta ; \underline{\underline{\nu}}, \underline{\lambda}}^{\mathrm{TV}}\left(q_{1}, \ldots, q_{L-1}, t\right)=$

$$
\begin{aligned}
& \mathbf{q}(\sigma, \theta ; \underline{v}, \underline{\lambda}) \cdot M(1, t)^{L} \cdot \prod_{j} s_{\lambda(j)}\left(t^{-\rho}\right) \cdot\left(\prod_{\alpha \in \Delta_{\underline{\text { re }},+}}\left(1+\sigma(\alpha) \mathbf{q}^{\alpha}\right)^{\sigma(\alpha) \alpha_{0}}\right) \\
& \begin{aligned}
\sum_{\nu_{-} \supseteq v_{1} \supseteq v_{2} \subseteq v_{3} \subseteq v_{+}} s_{\nu_{-} \backslash v_{1}}\left(S_{\sigma, \underline{\lambda}}^{+,-} ; \mathbf{q}\right) \cdot{ }^{\mathrm{t}} s_{v_{1} \backslash v_{2}}^{*}\left(S_{\sigma, \underline{\lambda}}^{-,-} ; \mathbf{q}\right) . \\
\left.s_{\nu_{3} \backslash v_{2}}\left(S_{\sigma, \underline{\lambda}}^{+,+} ; \mathbf{q}\right) \cdot{ }^{\mathrm{t}} s_{v_{+} \backslash v_{3}}^{*}\left(S_{\sigma, \underline{\lambda}}^{-,+} ; \mathbf{q}\right)\right) .
\end{aligned}
\end{aligned}
$$

Remark 4.17 In fact, we do not use non-commutative Donaldson-Thomas theory in the proof of this theorem.

\section{Construction of moduli spaces}

\subsection{Moduli space via a framed quiver with relations}

Since $A_{\sigma}^{\zeta}$ is Noetherian (see Lemma 1.4), we can take a presentation

$$
\oplus P_{i}^{\oplus b_{i}} \stackrel{J}{\rightarrow} \oplus P_{i}^{\oplus a_{i}} \rightarrow P_{\underline{\underline{\nu}}, \underline{\underline{\lambda}}}^{\zeta} \rightarrow 0
$$

of the finitely generate $A_{\sigma}^{\zeta}$-module $P_{\underline{v}, \underline{\lambda}}^{\zeta}$. Given a presentation, we define the new quiver with relation $\bar{A}_{\sigma}^{\xi}(\underline{v}, \underline{\lambda}):=\mathbb{C} \bar{Q} / \mathcal{J}$ as follows:

- the set of vertices of $\bar{Q}$ is given by $I \sqcup *$,

- the set of arrows of $\bar{Q}$ is given by adding $\iota_{a}\left(1 \leq a \leq a_{i}\right)$ from $*$ to $i$ for each $i$ to the set of arrows of $Q$, and

- the ideal $\mathcal{J}$ is generated by the relations of the original algebra $A_{\sigma}^{\zeta}$ and the elements of the following form:

$$
\sum \gamma_{a}(B) \cdot \iota_{a}
$$

for $B \in \oplus P_{i}^{\oplus b_{i}}$ and $J(B)=\sum \gamma_{a}(B) \cdot e_{a}$, where $e_{a} \in \oplus P_{i}^{\oplus a_{i}}$ is the idempotent in the direct summand corresponding to the index $a$.

Let $P_{*}$ (respectively $S_{*}$ ) be the projective (respectively simple) $\bar{A}_{\sigma}^{\zeta}$-module associated with the vertex $*$. The following claim is clear from the construction: 
Lemma 5.1 The kernel of the natural projection $P_{*} \rightarrow S_{*}$, as an $A_{\sigma}^{\zeta}$-module, is isomorphic to $P_{\underline{\underline{\nu}}, \underline{\underline{\lambda}}}^{\zeta}$.

According to this lemma, we have the natural isomorphism

$$
\begin{aligned}
\operatorname{Ext}_{\bar{A}_{\sigma}^{\xi}(\underline{v}, \underline{\lambda})}^{1}\left(S_{*}, V\right) & \simeq \operatorname{Hom}_{\bar{A}_{\sigma}^{\xi}(\underline{\underline{\nu}}, \underline{\lambda})}\left(\operatorname{Ker}\left(P_{*} \rightarrow S_{*}\right), V\right) \\
& \simeq \operatorname{Hom}_{A_{\sigma}^{\zeta}}\left(P_{\underline{\underline{v}}, \underline{\lambda}}^{\zeta}, V\right) .
\end{aligned}
$$

Moreover, $s \in \operatorname{Hom}_{A_{\sigma}^{\zeta}}\left(P_{\underline{v}, \underline{\lambda}}^{\zeta}, V\right)$ is surjective if and only if the $\bar{A}_{\sigma}^{\zeta}(\underline{v}, \underline{\lambda})$-module $V_{s}$ is generated by $\left(V_{S}\right)_{*}$, where $V_{S}$ is given by the extension

$$
0 \rightarrow V \rightarrow V_{s} \rightarrow S_{*} \rightarrow 0
$$

corresponding to $s$.

Take

$$
\begin{aligned}
\theta_{\text {cyc }} & \in\left(K_{\text {num }}\left(\bmod \bar{A}_{\sigma}^{\xi}(\underline{\nu}, \underline{\lambda})\right) \otimes \mathbb{R}\right)^{*} \\
& \simeq\left(K_{\text {num }}\left(\bmod A_{\sigma}^{\zeta}\right) \otimes \mathbb{R}\right)^{*} \oplus \mathbb{R}
\end{aligned}
$$

such that

$$
\theta_{\text {cyc }} \cdot(\mathbf{v}, 1)=0, \quad\left(\theta_{\text {cyc }}\right)_{i}>0(i \in I) .
$$

Then, the surjectivity condition above is equivalent to $\theta_{\text {cyc }}-$ stability. Hence we can construct the moduli space $\mathfrak{M}^{\text {ncDT }}(\zeta, \underline{v}, \underline{\lambda} ; \mathbf{v})$ as King's moduli space of $\theta_{\text {cyc }}$-stable $\bar{A}_{\sigma}^{\xi}(\underline{\underline{\nu}}, \underline{\lambda})$-modules with dimension vector $=(\mathbf{v}, 1) \in K_{\text {num }}\left(\bmod \bar{A}_{\sigma}^{\xi}(\underline{\underline{\nu}}, \underline{\lambda})\right) \simeq \mathbb{Z}^{I} \oplus \mathbb{Z}$.

Remark 5.2 For $\zeta \in\left(K_{\text {num }}\left(\bmod A_{\sigma}\right) \otimes \mathbb{R}\right)^{*}$, we can define the moduli space $\overline{\mathfrak{M}}_{A_{\sigma}}^{\zeta}(\mathbf{v})$ of $\zeta$-semistable framed $A_{\sigma}$-modules as in [27]. In [24], it is shown that

$$
\overline{\mathfrak{M}}_{A_{\sigma}}^{\zeta}(\mathbf{v}) \simeq \mathfrak{M}_{\bar{A}_{\sigma}^{\xi}(\underline{\varnothing}, \underline{ })}(\mathbf{v}, 1) \quad\left(\simeq \mathfrak{M}^{\mathrm{ncDT}}(\zeta, \underline{\varnothing}, \underline{\varnothing} ; \mathbf{v})\right) \text {. }
$$

\subsection{Moduli space via a framed quiver with a potential}

Assume $v=\underline{\varnothing}$. We put

$$
\begin{aligned}
\bigvee(\theta, \underline{\lambda}) & :=\{n \in \mathbb{Z} \mid \underline{\lambda} \circ \theta(n-1 / 2)=-, \underline{\lambda} \circ \theta(n+1 / 2)=+\} \\
& =\{n(1 / 2), \ldots, n(K+1 / 2)\}, \\
\bigwedge(\theta, \underline{\lambda}) & :=\{n \in \mathbb{Z} \mid \underline{\lambda} \circ \theta(n-1 / 2)=+, \underline{\lambda} \circ \theta(n+1 / 2)=-\} \\
& =\{n(1), \ldots, n(K)\},
\end{aligned}
$$


where $n(1 / 2)<n(1)<\cdots<n(K+1 / 2)$. We consider a newer quiver $\hat{Q}=\hat{Q}_{\sigma}^{\zeta}(\underline{\varnothing}, \underline{\lambda})$ obtained from $Q_{\sigma}^{\zeta}$ by adding

- an arrow $\iota_{a}$ from $*$ to $\pi(n(a))$ for $a=1 / 2, \ldots, K+1 / 2$, and

- an arrow $\tau_{b}$ from $\pi(n(b))$ to $*$ for $b=1, \ldots, K$.

We define a new potential $\widehat{w}=\widehat{w}_{\sigma}^{\zeta}(\underline{\varnothing}, \underline{\lambda})$ for the new quiver $\widehat{Q}$ by

$$
\widehat{w}:=w+\sum_{b=1}^{K}\left(\tau_{b} \circ h_{[n(b-1 / 2), n(b)]}{ }^{\circ} \iota_{b-1 / 2}-\tau_{b} \circ h_{[n(b+1 / 2), n(b)]}{ }^{\circ} \iota_{b+1 / 2}\right),
$$

where $w$ is the original potential and

$$
\begin{aligned}
& h_{[n(b-1 / 2), n(b)]}:=h_{\pi(n(b))-1 / 2}^{+} \circ \cdots \circ h_{\pi(n(b-1 / 2))+1 / 2}^{+}, \\
& h_{[n(b+1 / 2), n(b)]}:=h_{\pi(n(b))+1 / 2}^{-} \circ \cdots \circ h_{\pi(n(b+1 / 2))-1 / 2}^{-} .
\end{aligned}
$$

Let $\widehat{A}_{\sigma}^{\zeta}(\underline{\varnothing}, \underline{\lambda})$ be the Jacobi algebra of the $(\widehat{Q}, \widehat{w})$.

We take $\theta_{\text {cyc }}$ as in the previous subsection. Since the relations of $\widehat{A}_{\sigma}^{\zeta}(\underline{\varnothing}, \underline{\lambda})$ is obtained by the derivations of the potential, the moduli space

$$
\mathfrak{M}_{\widehat{A}_{\sigma}^{\zeta}(\underline{\varnothing}, \underline{\lambda})}^{\theta_{c y c}}(\mathbf{v}, 1)
$$

is the critical locus of a regular function and admits a symmetric obstruction theory.

We can show the following claim in the same way as in [27, Proposition 4.7].

\section{Proposition 5.3}

$$
\mathfrak{M}_{\bar{A}_{\sigma}^{\zeta}(\underline{\varnothing}, \underline{\lambda})}^{\theta_{\mathrm{cyc}}}(\mathbf{v}, 1) \simeq \mathfrak{M}_{\widehat{A}_{\sigma}^{\zeta}(\underline{\varnothing}, \underline{\lambda})}^{\theta_{\text {yc }}}(\mathbf{v}, 1)
$$

Combined with the result in the previous subsection, we see that $\mathfrak{M}^{\mathrm{ncDT}}(\zeta, \underline{\varnothing}, \underline{\lambda} ; \mathbf{v})$ admits a symmetric obstruction theory.

\section{Remarks and appendices}

\subsection{Weighted Euler characteristic}

Let $v: \mathfrak{M}^{\mathrm{ncDT}}(\zeta, \varnothing, \underline{\lambda} ; \mathbf{v}) \rightarrow \mathbb{Z}$ be the constructible function defined by Behrend [2] (the Behrend function). We define the generating function

$$
\mathcal{Z}_{\sigma, \zeta ; \underline{\varnothing}, \underline{\underline{\lambda}}}^{\mathrm{ncDT}}\left(q_{0}, \ldots, q_{L-1}\right):=\sum_{\mathbf{v}}\left(\sum_{n \in \mathbb{Z}} n \cdot \chi\left(v^{-1}(n)\right)\right) \cdot\left(\mathbf{q}_{\theta}\right)^{\mathbf{v}}
$$


where $\left(\mathbf{q}_{\theta}\right)^{\mathbf{v}}:=\prod\left(\mathbf{q}^{\alpha(\theta, i)}\right)^{v_{i}}$ as in Section 1.4. The Behrend function is defined for any scheme over $\mathbb{C}$. In [2], Behrend showed that if a proper scheme has a symmetric obstruction theory then the virtual counting, which is defined by integrating the constant function 1 over the virtual fundamental cycle, coincides with the weighted Euler characteristic weighted by the Behrend function as above. Based on this result, he proposed to define the virtual counting for a non-proper variety with a symmetric obstruction theory as the weighted Euler characteristic.

We can apply Behrend-Fantechi's result [4, Theorem 3.4] to compute the weighted Euler characteristic by torus localization. The contribution of a torus fixed point is +1 or -1 , which is determined by the parity of the dimension of the Zariski tangent space. Since we have a description of $\mathfrak{M}^{\text {ncDT }}(\zeta, \underline{\varnothing}, \underline{\lambda} ; \mathbf{v})$ as the moduli space of $\widehat{A}_{\sigma}^{\xi}(\underline{\varnothing}, \underline{\lambda})$-modules in Section 5.2, the dimension of the Zariski tangent space at an $\widehat{A}_{\sigma}^{\zeta}(\underline{\varnothing}, \underline{\underline{\lambda}})$-module $M$ is given by

$$
1-\operatorname{hom}(M, M)+\operatorname{ext}^{1}(M, M)
$$

(note that 1 corresponds to the identity morphism of $M$ ). It is shown by Joyce and Song [20, Equation (140)] that the first (respectively second) cohomology of the following complex is $\operatorname{Hom}(M, M)$ (respectively $\left.\operatorname{Ext}^{1}(M, M)\right)$ :

$$
0 \rightarrow \bigoplus_{i \in \hat{Q}_{0}} M_{i}^{*} \otimes M_{i} \rightarrow \bigoplus_{e \in \widehat{Q}_{1}} M_{t(e)}^{*} \otimes M_{h(e)} \rightarrow \bigoplus_{e \in \hat{Q}_{1}} M_{h(e)}^{*} \otimes M_{t(e)}
$$

Note that this is an analogue of the Kozsul complex.

Since the quiver with potential $(Q, W)$ is induced by a consistent brane tiling, we have a $\Lambda_{Q, W}$-grading for $Q$ for a free abelian group $\Lambda_{Q, W}$ such that the potential is homogeneous of degree $d \in \Lambda_{Q, W}$ where $d$ is not divisible by 2 (see Mozgovoy and Reineke [23, Section 3]). In Section 5.2, we construct the new quiver $\hat{Q}=\hat{Q}_{\sigma}^{\zeta}(\underline{\varnothing}, \underline{\lambda})$ by adding $2 K+1$ arrows and the new potential $\widehat{w}=\widehat{w}_{\sigma}^{\zeta}(\underline{\varnothing}, \underline{\lambda})$ by adding $K$ terms to the original homogeneous potential. We can extend the $\Lambda_{Q, W}$-grading to $\hat{Q}$ so that $\widehat{w}$ is homogeneous of degree $d$. Then, by the same argument as [23, Theorem 7.1], the parity of (6-1) coincides with the parity of the difference of the dimensions of the first two terms of the complex (6-2) plus 1.

For an $\widehat{A}_{\sigma}^{\zeta}(\underline{\varnothing}, \underline{\lambda})$-module $M$ with the dimension vector $\widetilde{\mathbf{v}}=(\mathbf{v}, 1)$, the difference of the dimensions of the first two terms of the complex (6-2) plus 1 is given by

$$
\begin{aligned}
& 1-\sum_{i \in \widehat{Q}_{0}} \tilde{\mathbf{v}}_{i}^{2}+\sum_{e \in \widehat{Q}_{1}} \tilde{\mathbf{v}}_{t(e)} \tilde{\mathbf{v}}_{h(e)}= \\
& \quad-\sum_{i \in Q_{0}} \mathbf{v}_{i}^{2}+\sum_{i \in Q_{0}} Q_{i \rightarrow i} \cdot \mathbf{v}_{i}^{2}+\sum_{i \neq j \in Q_{0}}\left(Q_{i \rightarrow j}+Q_{j \rightarrow i}\right) \mathbf{v}_{i} \mathbf{v}_{j}+\sum_{i \in Q_{0}}\left(\hat{Q}_{i \rightarrow \bullet}+\hat{Q}_{\bullet \rightarrow i}\right) \mathbf{v}_{i}
\end{aligned}
$$


where $Q_{i \rightarrow j}$ (respectively $\widehat{Q}_{i \rightarrow j}$ ) is the number of arrows in the quiver $Q$ (respectively $\hat{Q}$ ) from the vertex $i$ to the vertex $j$. Since we have $Q_{i \rightarrow j}=Q_{j \rightarrow i}$, the parity is given by

$$
\equiv \sum_{i \in Q_{0}}\left(1+Q_{i \rightarrow i}+\hat{Q}_{i \rightarrow \bullet}+\hat{Q}_{\bullet \rightarrow i}\right) \mathbf{v}_{i}
$$

As a result we get

$$
\mathcal{Z}_{\sigma, \zeta ; \underline{\varnothing}, \underline{\lambda}}^{\mathrm{ncDT}}\left(q_{0}, \ldots, q_{L-1}\right)=\mathcal{Z}_{\sigma, \zeta ; \underline{\varnothing}, \underline{\lambda}}^{\mathrm{E}-\mathrm{ncDT}}\left(p_{0}, \ldots, p_{L-1}\right)
$$

under the variable change given by

$$
\mathbf{p}^{\alpha(\theta, i)}=(-1)^{1+Q_{i \rightarrow i}+\widehat{Q}_{i \rightarrow *}+\widehat{Q}_{* \rightarrow i}} \mathbf{q}^{\alpha(\theta, i)} .
$$

\subsection{Pandharipande-Thomas invariants and wall-crossing}

As we mentioned at the end of Section 1.3, we have worked on the area $\{\zeta \mid \zeta \cdot \delta<0\}$ up to now. In this subsection, we make some comments on the area $\{\zeta \mid \zeta \cdot \delta>0\}$, that is, $0<t<1 / 2$. We have a natural bijection between $\Theta$ and the set of chambers in the area $\{\zeta \mid \zeta \cdot \delta>0\}$ as well. An element $\zeta_{\theta}$ in the chamber $C^{\theta}$ corresponding to $\theta \in \Theta$ satisfies the following condition:

$$
\alpha_{\left[h, h^{\prime}\right]} \cdot \zeta_{\theta}>0 \Longleftrightarrow \theta(h)<\theta\left(h^{\prime}\right)
$$

for any $h<h^{\prime}$.

Note that $\mathcal{D}_{\text {fin }}^{\zeta^{\circ}}[t, 0)$ is a left admissible full subcategory of $D^{b}\left(\bmod A_{\sigma}\right)$ for $0<t<1$. Let ${ }^{\mathrm{t}} \mathcal{D}_{\text {fin }}^{\zeta^{\circ}}[t+1, t)$ denote the core of t-structure given from $\bmod A_{\sigma}$ by tilting with respect to $\mathcal{D}_{\text {fin }}^{\zeta^{\circ}}\left[t, 0\right.$ ) (this is different from $\mathcal{D}^{\zeta^{\circ}}[t, t-1)[1]$ given in Section 1.2) and we put

$$
{ }^{\mathrm{t}} \mathcal{A}^{\zeta}:={ }^{\mathrm{t}} \mathcal{D}_{\text {fin }}^{\zeta^{\circ}}[t+1, t)[-1] .
$$

(see Definition 1.7). As in Proposition 1.10 we have

$$
{ }^{\mathrm{t}} \mathcal{A}^{\zeta} \simeq \bmod _{\mathrm{fin}} A_{\sigma}^{\zeta} .
$$

We put

$$
{ }^{\mathrm{t}} P_{\underline{\underline{v}, \underline{\lambda}}}^{\zeta}:=H_{\mathrm{t} \mathcal{A}^{\zeta}}^{0}\left(\mathcal{I}_{\underline{\nu}, \underline{\lambda}}\right)
$$

(see (2-1)) and let $\mathfrak{M}^{\text {ncPT }}(\zeta, \underline{v}, \underline{\lambda} ; \mathbf{v})$ denote the moduli space of quotient objects $V$ of ${ }^{\mathrm{t}} P_{\underline{\underline{v}}, \underline{\lambda}}^{\zeta}$ in ${ }^{\mathrm{t}} \mathcal{A}^{\zeta}$ with $[V]=\mathbf{v}$ : (see Definition 2.2 and Theorem 2.3).

Example 6.1 Assume that $L_{+}=L_{-}=1 \underline{\nu}=\varnothing$ and $\underline{\lambda}=\varnothing$. Take $\zeta^{m .+} \in \mathbb{Z}^{2}$ such that

$$
\zeta_{0}^{m,+}<\zeta_{1}^{m,+}, \quad m \zeta_{0}^{m,+}+(m-1) \zeta_{1}^{m,+}<0, \quad(m+1) \zeta_{0}^{m,+}+m \zeta_{1}^{m,+}>0 .
$$


as in [27, Section 4.1]. Then we have

$$
P_{\underline{\varnothing} . \underline{\varnothing}}^{\xi^{m,+}}=\mathcal{O}_{C}^{(m, m-1, \ldots, 1)}
$$

where $\mathcal{O}_{C}^{(m, m-1, \ldots, 1)}$ is given by the exact sequence

$$
0 \rightarrow \mathcal{I}(\underline{\varnothing}, \underline{\varnothing} ;(m, m-1, \ldots, 1)) \rightarrow \mathcal{O}_{Y_{\sigma}} \rightarrow \mathcal{O}_{C}^{(m, m-1, \ldots, 1)} \rightarrow 0 .
$$

As an $A_{\sigma \circ \theta}$-module, $\mathcal{O}_{C}^{(m, m-1, \ldots, 1)}$ is given by "the finite type pyramid with length $m$ " (see Chuang and Pan [13]). Hence the moduli $\mathfrak{M}^{\text {ncPT }}\left(\zeta^{m,+}, \underline{\varnothing}, \underline{\varnothing} ; \mathbf{v}\right)$ coincides with the one we denoted by $\mathfrak{M}_{\xi_{\text {cyclic }}}^{A}(\mathbf{v})$ in [27, Section 4].

Take the limit of $\theta$, the following moduli appears:

$$
\left\{s: \mathcal{I}_{\underline{v}, \underline{\lambda}} \rightarrow F \mid F: \text { pure of dimension } 1, \operatorname{dim} \operatorname{ker}(s)=1\right\} .
$$

This is a generalization of the moduli of stable pairs in the sense of PandharipandeThomas [31]. Thus let us temporarily define the Euler characteristic of the moduli space $\mathfrak{M}^{\text {ncPT }}(\zeta, \underline{\nu}, \underline{\lambda} ; \mathbf{v})$ as the open non-commutative Pandharipande-Thomas (open ncPT in short) invariant.

On the other hand, we define the finite type transition of Young diagrams (and hence the finite type crystal model) of type $(\sigma, \theta ; \underline{\nu}, \underline{\lambda})$ by replacing $\theta$ by $(-1) \circ \theta$, where $(-1): \mathbb{Z}_{\mathrm{h}} \rightarrow \mathbb{Z}_{\mathrm{h}}$ is the multiplication of $(-1)$. The generating function is described by the operator

$$
\prod_{h \in \mathbb{Z}_{\mathrm{h}}}^{(-1) \circ \theta} \Gamma_{\lambda(h)}^{\sigma(h)}\left(q_{h}\right)
$$

and we can compute it in the same way as Section $3.3^{8}$.

The author expects that as an $A_{\sigma}^{\zeta}$-module ${ }^{\mathrm{t}} P_{\underline{\underline{v}}, \underline{\lambda}}^{\zeta}$ is given by the grand state finite type crystal and hence we can compute the generating function of open ncPT invariants explicitly. We can check this is true for some concrete examples (see Example 6.1). If this is true in general,

- we get open version of DT-PT correspondence in our setting, and

- we can realize the normalized generating function appearing in Corollary 3.22 as the generating function of Euler characteristics of the moduli spaces

$$
\mathfrak{M}^{\text {ncPT }}\left(\zeta^{\text {triv }}, \underline{\nu}, \underline{\lambda} ; \mathbf{v}\right)
$$

for $\zeta^{\text {triv }} \in \mathbb{R}^{I}$ such that $\left(\zeta^{\text {triv }}\right)_{i}>0$ for any $i$.

${ }^{8} \mathrm{~A}$ similar crystal melting model and computation are given by Sulkowski [32]. 


\subsection{Proof of Proposition 3.7}

Lemma 6.2 (Young [12, Lemma 3.2]) For two Young diagrams $\mu$ and $\mu^{\prime}, \mu \stackrel{+}{\succ} \mu^{\prime}$ if and only if ${ }^{\mathrm{t}} \mu_{c}-{ }^{\mathrm{t}} \mu_{c}^{\prime}=0$ or 1 for any $c \in \mathbb{Z}_{\geq 0}$.

Note that $\mathcal{V}_{\min }(n)$ is determined by $\mathcal{V}_{\min }(n-1)$ and $\mathcal{V}_{\min }(n+1)$

Example 6.3 Assume that

$$
\sigma \circ \theta(n-1 / 2)=\sigma \circ \theta(n+1 / 2)=\underline{\lambda} \circ \theta(n-1 / 2)=\underline{\lambda} \circ \theta(n+1 / 2)=+.
$$

Then we have ${ }^{\mathrm{t}} \mathcal{V}(n-1)_{c}-{ }^{\mathrm{t}} \mathcal{V}(n+1)_{c}=0,1$ or 2 for any transition $\mathcal{V}$ and

$$
{ }^{\mathrm{t}} \mathcal{V}_{\min }(n-1)_{c}-{ }^{\mathrm{t}} \mathcal{V}_{\min }(n)_{c}= \begin{cases}0 & { }^{\mathrm{t}} \mathcal{V}_{\min }(n-1)_{c}-{ }^{\mathrm{t}} \mathcal{V}_{\min }(n+1)_{c}=0, \\ 1 & { }^{\mathrm{t}} \mathcal{V}_{\min }(n-1)_{c}-{ }^{\mathrm{t}} \mathcal{V}_{\min }(n+1)_{c}=1 \text { or } 2\end{cases}
$$

For a transition $\mathcal{V}$ of Young diagram, an addable $i$-node for $\mathcal{V}$ is an element $(n, x, y)$ such that $\pi(n)=i,(x, y) \neq \mathcal{V}(n)$ and

$$
\mathcal{V}^{\prime}(m):= \begin{cases}\mathcal{V}(m) & m \neq n, \\ \mathcal{V}(n) \sqcup(x, y) & m=n,\end{cases}
$$

gives a transition. Let $\mathcal{V}^{[i]}$ denote the transition given by adding all addable $i$-node for $\mathcal{V}$.

Example 6.4 Assume that

$$
\sigma \circ \theta(n-1 / 2)=\sigma \circ \theta(n+1 / 2)=\underline{\lambda} \circ \theta(n-1 / 2)=\underline{\lambda} \circ \theta(n+1 / 2)=+.
$$

Then $\mathcal{V}^{[i]}(n \pm 1)=\mathcal{V}(n \pm 1)$ and

$$
{ }^{\mathrm{t}} \mathcal{V}_{\min }(n-1)_{c}-{ }^{\mathrm{t}} \mathcal{V}_{\min }(n)_{c}= \begin{cases}0 & { }^{\mathrm{t}} \mathcal{V}_{\min }(n-1)_{c}-{ }^{\mathrm{t}} \mathcal{V}_{\min }(n+1)_{c}=0 \text { or } 1 \\ 1 & { }^{\mathrm{t}} \mathcal{V}_{\min }(n-1)_{c}-{ }^{\mathrm{t}} \mathcal{V}_{\min }(n+1)_{c}=1\end{cases}
$$

For a transition $\mathcal{V}, M\left(\mathcal{V}^{[i]}\right)$ does not have the simple module $S_{i}$ as its quotient. Note that for an $A_{\sigma}$-module $M$ without the simple module $S_{i}$ as its quotient, $M$ is identified with an $A_{\sigma \circ \theta \circ \theta_{i}}-$ module $\mu_{i} M$ in $D^{b}\left(\bmod A_{\sigma}\right)$, and

$$
\left(\mu_{i} M\right)_{i^{\prime}}= \begin{cases}M_{k} & k \neq i, \\ \operatorname{ker}\left(M_{i-1} \oplus M_{i+1} \rightarrow M_{i}\right) & k=i .\end{cases}
$$

Let $\mathcal{V}_{\min }^{\prime}:=\mathcal{V}_{\min }^{\sigma, \theta \circ \theta_{i}, \underline{v}, \underline{\lambda}}$ be the minimal transition of type $\left(\sigma, \theta \circ \theta_{i}, \underline{v}, \underline{\lambda}\right)$. 
Proposition 6.5 As $A_{\sigma \circ \theta \circ \theta_{i}}$-modules, $\mu_{i} M\left(\mathcal{V}_{\min }^{[i]}\right)$ is isomorphic to $M\left(\mathcal{V}_{\min }^{\prime}\right)$.

Proof Note that $\mathcal{V}_{\text {min }}(n)=\mathcal{V}_{\text {min }}^{\prime}(n)$ if $\pi(n) \neq i$. We will define isomorphisms

$$
\operatorname{ker}\left(M\left(\mathcal{V}_{\text {min }}\right)_{n-1} \oplus M\left(\mathcal{V}_{\text {min }}\right)_{n+1} \rightarrow M\left(\mathcal{V}_{\text {min }}\right)_{n}\right) \stackrel{\sim}{\longrightarrow} M\left(\mathcal{V}_{\text {min }}^{\prime}\right)_{n}
$$

for $n$ such that $\pi(n)=i$. For example, assume that

$$
\sigma \circ \theta(n-1 / 2)=\sigma \circ \theta(n+1 / 2)=\underline{\lambda} \circ \theta(n-1 / 2)=\underline{\lambda} \circ \theta(n+1 / 2)=+.
$$

Note that $\mathcal{V}_{\min }=\mathcal{V}_{\min }^{\prime}$ in this case. For $n$ such that $\pi(n)=i$,

$$
\operatorname{ker}\left(M\left(\mathcal{V}_{\min }^{[i]}\right)_{n-1} \oplus M\left(\mathcal{V}_{\min }^{[i]}\right)_{n+1} \rightarrow M\left(\mathcal{V}_{\min }^{[i]}\right)_{n}\right)
$$

is spanned by the following elements:

$$
\begin{array}{lr}
-p\left(n+1, x,{ }^{\mathrm{t}} \mathcal{V}_{\min }(n)_{x}\right) & { }^{\mathrm{t}} \mathcal{V}_{\min }(n-1)_{x}-{ }^{\mathrm{t}} \mathcal{V}_{\min }(n+1)_{x}=0, \\
p(n-1, x, y)-p(n+1, x, y-1) & { }^{\mathrm{t}} \mathcal{V}_{\min }(n-1)_{x}-{ }^{\mathrm{t}} \mathcal{V}_{\min }(n+1)_{x}=0, \\
p(n-1, x, y)-p(n+1, x, y-1) & \text { and } y=2, \ldots,{ }^{\mathrm{t}} \mathcal{V}_{\min }(n)_{x}, \\
& { }^{\mathrm{t}} \mathcal{V}_{\min }(n-1)_{x}-{ }^{\mathrm{t}} \mathcal{V}_{\min }(n+1)_{x}=1, \\
p\left(n-1, x,{ }^{\mathrm{t}} \mathcal{V}_{\min }(n)_{x}+1\right) & \text { and } y=2, \ldots,{ }^{\mathrm{t}} \mathcal{V}_{\min }(n)_{x}+1, \\
p(n-1, x, y)-p(n+1, x, y-1) & { }^{\mathrm{t}} \mathcal{V}_{\min }(n-1)_{x}-{ }^{\mathrm{t}} \mathcal{V}_{\min }(n-1)_{x}-{ }^{\mathrm{t}} \mathcal{V}_{\min }(n+1)_{x}=2, \\
& \text { and } y=2, \ldots,{ }^{\mathrm{t}} \mathcal{V}_{\min }(n)_{x} .
\end{array}
$$

The isomorphism is given by mapping one of elements above involving $p(n-1, x, y)$ or $p(n+1, x, y-1)$ to $p^{\prime}(n, x, y)$. We can verify this isomorphism respects the actions of $A_{\sigma \circ \theta \circ \theta_{i}}$.

Proof of Proposition 3.7 This proof consists of four steps.

Step 1 In the case $\theta=\mathrm{id}, \underline{v}=\underline{\varnothing}$ and $\underline{\lambda}=\underline{\varnothing}$, we have $\mathcal{V}_{\min }^{\sigma, \mathrm{id}, \underline{\varnothing}, \underline{\varnothing}}(n)=\varnothing$ for any $n$ and

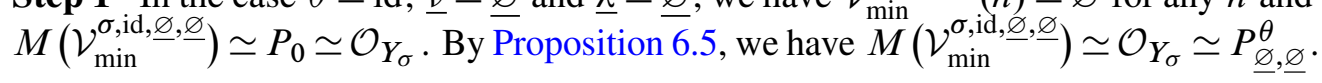

Step 2 The following is shown in [26, Theorem 5.8]:

Given $\beta$ and $n$, we can take sufficiently small $T>0$ such that giving a crystal of type $(\sigma, \theta ; \underline{v}, \underline{\lambda})$ is equivalent to giving a data $(\vec{v}, \vec{\Lambda})$ as in Section 4.2. 
More precisely, we have $L$ intervals $C_{1 / 2}, \ldots, C_{L-1 / 2}$ disjoint with each other such that in the interval $C_{j}$

$$
\bigsqcup_{n \in C_{j}} \mathcal{V}(n)
$$

is identified with the 3-dimensional Young diagram $\Lambda^{(j)}$. Here, a box in the diagram $\Lambda^{(j)}$ is identified with an $L$-tuple of elements from successive Young diagrams $\mathcal{V}(n), \ldots, \mathcal{V}(n+L-1)$ for some $n$, which is isomorphic to the skyscraper sheaf of the $j$ th fixed point in $Y_{\sigma}$ as an $A_{\sigma \circ \theta}$-module.

Step 3 Take sufficiently small $T>0$, such that

$$
0 \rightarrow \mathcal{O}_{Z_{\underline{\underline{\nu}}, \underline{\lambda}}} \rightarrow \mathcal{O}_{Y_{\sigma}} \rightarrow \mathcal{I}_{\underline{\underline{\nu}}, \underline{\lambda}} \rightarrow 0
$$

is an exact sequence is $\mathcal{A}_{\text {fin }}^{\zeta}$ for $\zeta=\zeta_{\text {cyc }}^{\circ}+T \eta$. Note that the closed subscheme $Z_{\underline{v}, \underline{\lambda}} \subset Y_{\sigma}$ is decomposed into the disjoint union of closed subschemes

$$
Z_{\underline{v}, \underline{\lambda}}^{(j)} \subset U^{(j)} \subset Y_{\sigma}
$$

where $U^{(j)}$ is the toric coordinate locus around the $j$ th fixed point.

Hence, what we have to show is

$$
\bigoplus_{n \in C_{j},(x, y) \in \mathcal{V}(n)} \mathbb{C} \cdot(n, x, y)
$$

is isomorphic to $\mathcal{O}_{\underline{\nu}, \underline{\lambda}}^{(j)}:=\mathcal{O}_{Z_{\underline{\nu}, \underline{\lambda}}^{(j)}}$ as an $A_{\sigma \circ \theta}$-module.

Step 4 In [24, Proposition 3.5], it is shown that the derived equivalence $\Phi$ between $Y_{\sigma}$ and $A_{\sigma \circ \theta}$ is given by a tilting vector bundle on $Y_{\sigma}$ which is a direct sum of line bundles. Hence $\Phi\left(\mathcal{O}_{\underline{\underline{\nu}}, \underline{\underline{\lambda}}}^{(j)}\right)_{i}$ is isomorphic to $\mathcal{O}_{\underline{\underline{v}}, \underline{\underline{\lambda}}}^{(j)}$, and hence to

$$
\bigoplus_{\substack{n \in C_{j}, \pi(n)=i,(x, y) \in \mathcal{V}(n)}} \mathbb{C} \cdot(n, x, y)
$$

as $\mathcal{O}_{X}$-modules for any $i$.

Moreover, in [24, Proposition 3.5] we described the map between line bundles corresponding to the arrow $h_{k}^{ \pm}$in the quiver, which induces an endomorphisms on $\mathcal{O}_{\underline{\underline{\nu}}, \underline{\lambda}}^{(j)}$. We can check this endomorphism coincides with

$$
\bigoplus_{\substack{n \in C_{j}, \pi(n)=k \mp 1 / 2,(x, y) \in \mathcal{V}(n)}} \mathbb{C} \cdot(n, x, y) \rightarrow \bigoplus_{\substack{n \in C_{j}, \pi(n)=k \pm 1 / 2,(x, y) \in \mathcal{V}(n)}} \mathbb{C} \cdot(n, x, y)
$$

given by the Definition 3.6. Hence Proposition 3.7 follows. 
Example 6.6 In Figure 2, 3 and 4, we provide some examples which may help the reader to understand the proof. All the examples are in the case of $L_{+}=L_{-}=1$, that is, the conifold case. In Figure 2 we provide the figure of a part of the grand state crystal in the case of $\underline{v}=\varnothing$ and $\underline{\lambda}=\varnothing$. In Figure 3 and 4 we provide the figures of grand state and

$$
\bigsqcup_{n \in C_{3 / 2}} \mathcal{V}(n)
$$

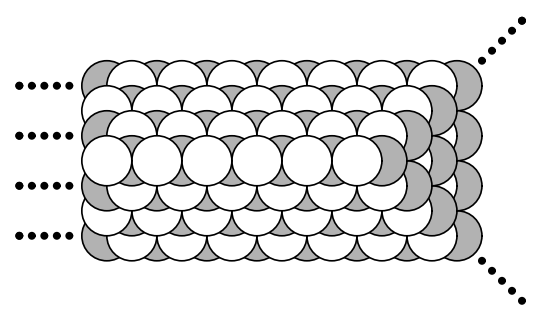

Figure 2: $\underline{v}=\underline{\varnothing}$ and $\underline{\lambda}=\underline{\varnothing}$.
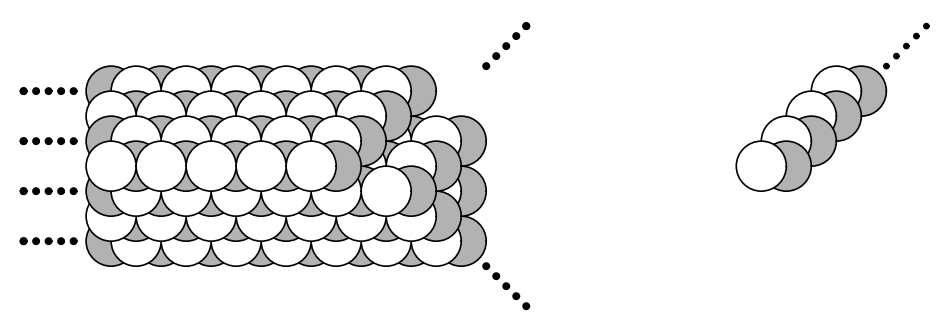

Figure 3: $\underline{v}=\underline{\varnothing}$ and $\underline{\lambda}=(\varnothing, \square)$
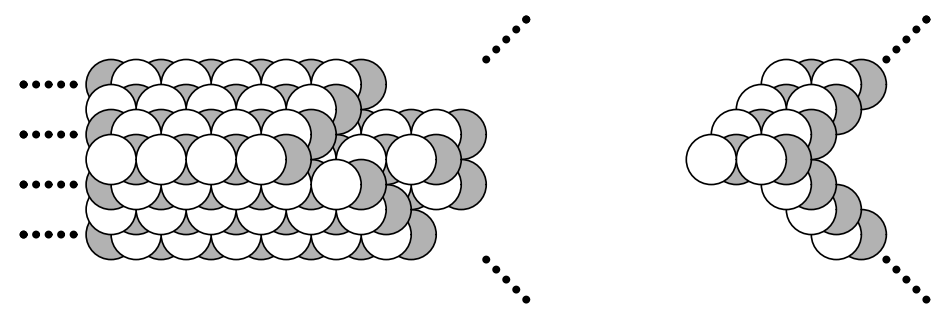

Figure 4: $\underline{v}=(\varnothing, \square)$ and $\underline{\lambda}=(\varnothing, \square)$ 


\section{References}

[1] M Aganagic, A Klemm, M Mariño, C Vafa, The topological vertex, Comm. Math. Phys. 254 (2005) 425-478 MR2117633

[2] K Behrend, Donaldson-Thomas type invariants via microlocal geometry, Ann. of Math. (2) 170 (2009) 1307-1338 MR2600874

[3] K Behrend, B Fantechi, The intrinsic normal cone, Invent. Math. 128 (1997) 45-88 MR1437495

[4] K Behrend, B Fantechi, Symmetric obstruction theories and Hilbert schemes of points on threefolds, Algebra Number Theory 2 (2008) 313-345 MR2407118

[5] M van den Bergh, Non-commutative crepant resolutions arXiv:math/0211064

[6] M van den Bergh, Three-dimensional flops and noncommutative rings, Duke Math. J. 122 (2004) 423-455 MR2057015

[7] R Bocklandt, Calabi-Yau algebras and weighted quiver polyhedra arXiv: 0905.0232

[8] T Bridgeland, $t$-structures on some local Calabi-Yau varieties, J. Algebra 289 (2005) 453-483 MR2142382

[9] T Bridgeland, Stability conditions on triangulated categories, Ann. of Math. (2) 166 (2007) 317-345 MR2373143

[10] N Broomhead, Dimer models and Calabi-Yau algebras arXiv:0901.4662

[11] J Bryan, C Cadman, B Young, The orbifold topological vertex arXiv:1008.4205

[12] J Bryan, B Young, Generating functions for colored 3D Young diagrams and the Donaldson-Thomas invariants of orbifolds, Duke Math. J. 152 (2010) 115-153 MR2643058 With an appendix by Jim Bryan

[13] W-Y Chuang, G Pan, Bogomolny-Prasad-Sommerfeld state counting in local obstructed curves from quiver theory and Seiberg duality, J. Math. Phys. 51 (2010) 052305, 22 MR2666961

[14] B Davison, Consistency conditions for brane tilings arXiv:0812.4185

[15] A Hanany, D Vegh, Quivers, tilings, branes and rhombi, J. High Energy Phys. (2007) 029, 35 MR2357949

[16] D Happel, I Reiten, S O Smalø, Tilting in abelian categories and quasitilted algebras, Mem. Amer. Math. Soc. 120 (1996) MR1327209

[17] A Ishii, K Ueda, Dimer models and the special McKay correspondence arXiv: 0905.0059

[18] A Ishii, K Ueda, On moduli spaces of quiver representations associated with dimer models arXiv:0710.1898

[19] D Joyce, Configurations in abelian categories IV: Invariants and changing stability conditions, Adv. Math. 217 (2008) 125-204 MR2357325 
[20] D Joyce, Y Song, A theory of generalzed Donaldson-Thomas invariants arXiv: 0810.5645

[21] D Maulik, N Nekrasov, A Okounkov, R Pandharipande, Gromov-Witten theory and Donaldson-Thomas theory I, Compos. Math. 142 (2006) 1263-1285 MR2264664

[22] S Mozgovoy, Crepant resolutions and brane tilings I: Toric realization arXiv: 0908.3475

[23] S Mozgovoy, M Reineke, On the noncommutative Donaldson-Thomas invariants arising from brane tilings, Adv. Math. 223 (2010) 1521-1544 MR2592501

[24] K Nagao, Derived categories of small 3-dimensional toric Calabi-Yau varieties and curve counting invariants arXiv:0809.2994

[25] K Nagao, Quiver varieties and Frenkel-Kac construction, J. Algebra 321 (2009) 37643789 MR2517812

[26] K Nagao, Refined open non-commutative Donaldson-Thomas theory for small toric Calabi-Yau 3-folds, Pacific J. Math. (to appear) arXiv:0907.3784

[27] K Nagao, H Nakajima, Counting invariants of perverse coherent systems on 3-folds and their wall-crossings, Int. Math. Res. Not. 46 (2010)

[28] K Nagao, M Yamazaki, The non-commutative topological vertex and wall crossing phenomena (to appear) arXiv:0910.5479

[29] A Okounkov, N Reshetikhin, C Vafa, Quantum Calabi-Yau and classical crystals, from: “The unity of mathematics”, Progr. Math. 244, Birkhäuser, Boston (2006) 597618 MR2181817

[30] H Ooguri, M Yamazaki, Crystal melting and toric Calabi-Yau manifolds, Comm. Math. Phys. 292 (2009) 179-199 MR2540074

[31] R Pandharipande, R P Thomas, Curve counting via stable pairs in the derived category, Invent. Math. 178 (2009) 407-447 MR2545686

[32] P Sułkowski, Wall-crossing, free fermions and crystal melting, Comm. Math. Phys. 301 (2011) 517-562 MR2764996

[33] B Szendrói, Non-commutative Donaldson-Thomas invariants and the conifold, Geom. Topol. 12 (2008) 1171-1202 MR2403807

[34] B Young, Computing Donaldson-Thomas partition functions for brane tilings using vertex operators (to appear)

RIMS, Kyoto University

Kyoto 606-8502, Japan

kentaron@kurims.kyoto-u.ac.jp

Proposed: Jim Bryan

Seconded: Richard Thomas, Simon Donaldson

Received: 17 November 2009

Revised: 23 April 2011 